\title{
Submillimeter line emission from LMC 30 Doradus: The impact of a starburst on a low-metallicity environment
}

\author{
J. L. Pineda ${ }^{1}$, N. Mizuno ${ }^{2}$, M. Röllig ${ }^{3}$, J. Stutzki ${ }^{3}$, C. Kramer ${ }^{4}$, U. Klein ${ }^{5}$, M. Rubio ${ }^{6}$, A. Kawamura ${ }^{2}$, T. Minamidani ${ }^{7}$, \\ A. Benz ${ }^{8}$, M. Burton ${ }^{9}$, Y. Fukui ${ }^{10}$ B.-C. Koo ${ }^{11}$, and T. Onishi ${ }^{12}$
}

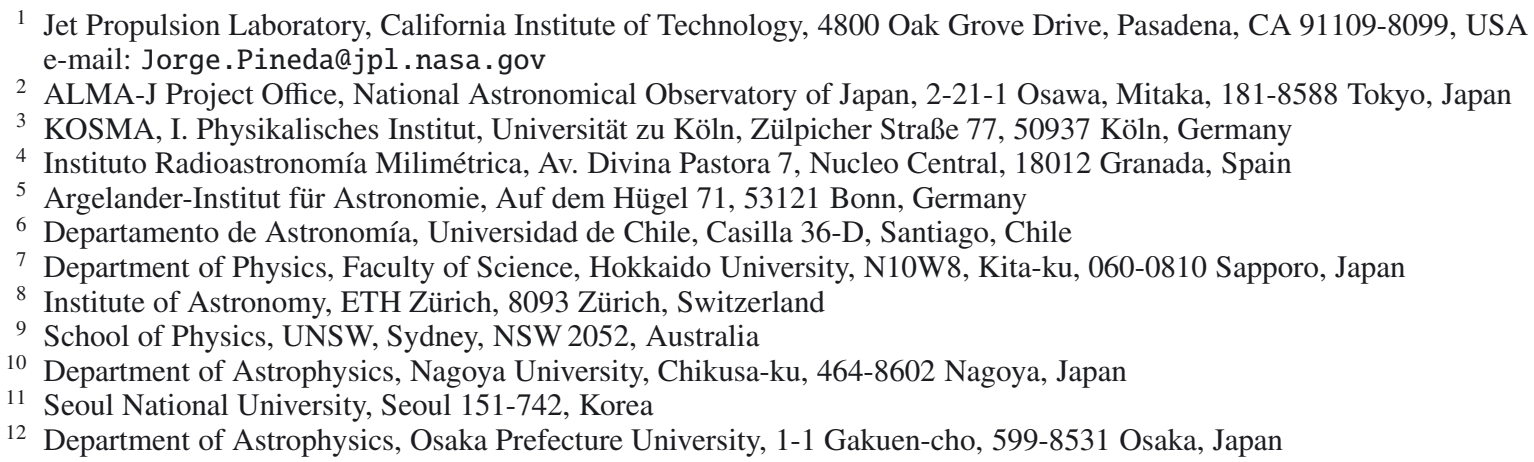

Received 22 October 2011 / Accepted 15 June 2012

\begin{abstract}
Context. The 30 Dor region in the Large Magellanic Cloud (LMC) is the most vigorous star-forming region in the Local Group. Star formation in this region is taking place in low-metallicity molecular gas that is exposed to an extreme far-ultraviolet (FUV) radiation field powered by the massive compact star cluster R136. 30 Dor is therefore ideally suited to study the conditions in which stars formed at earlier cosmological times.

Aims. Observations of (sub)mm and far-infrared (FIR) spectral lines of the main carbon-carrying species, $\mathrm{CO},\left[\mathrm{C}_{\mathrm{I}}\right]$ and $\left[\mathrm{C}_{\mathrm{II}}\right]$, which originate in the surface layers of molecular clouds illuminated by the FUV radiation of young stars, can be used to constrain the physical and chemical state of the star-forming ISM.

Methods. We used the NANTEN2 telescope to obtain high-angular resolution observations of the ${ }^{12} \mathrm{CO} J=4 \rightarrow 3, J=7 \rightarrow 6$, and ${ }^{13} \mathrm{CO} J=4 \rightarrow 3$ rotational lines and $\left[\mathrm{C}_{\mathrm{I}}\right]{ }^{3} \mathrm{P}_{1}-{ }^{3} \mathrm{P}_{0}$ and ${ }^{3} \mathrm{P}_{2}-{ }^{3} \mathrm{P}_{1}$ fine-structure submillimeter transitions in 30 Dor-10, the brightest $\mathrm{CO}$ and FIR-emitting cloud at the center of the 30 Dor region. We derived the physical and chemical properties of the low-metallicity molecular gas using an excitation/radiative transfer code and found a self-consistent solution of the chemistry and thermal balance of the gas in the framework of a clumpy cloud PDR model. We compared the derived properties with those in the N159W region, which is exposed to a more moderate far-ultraviolet radiation field compared with 30 Dor-10, but has similar metallicity. We also combined our $\mathrm{CO}$ detections with previously observed low- $J \mathrm{CO}$ transitions to derive the CO spectral-line energy distribution in $30 \mathrm{Dor}-10$ and $\mathrm{N} 159 \mathrm{~W}$.

Results. The separate excitation analysis of the submm CO lines and the neutral carbon fine structure lines shows that the mid- $J$ $\mathrm{CO}$ and $\left[\mathrm{C}_{\mathrm{I}}\right]$-emitting gas in the 30 Dor-10 region has a temperature of about $160 \mathrm{~K}$ and a $\mathrm{H}_{2}$ density of about $10^{4} \mathrm{~cm}^{-3}$. We find that the molecular gas in 30 Dor-10 is warmer and has a lower beam filling factor compared to that of N159W, which might be a result of the effect of a strong FUV radiation field heating and disrupting the low-metallicity molecular gas. We use a clumpy PDR model (including the $\left[\mathrm{C}_{\mathrm{II}}\right]$ line intensity reported in the literature) to constrain the FUV intensity to about $\chi_{0} \approx 3100$ and an average total $\mathrm{H}$ density of the clump ensemble of about $10^{5} \mathrm{~cm}^{-3}$ in 30 Dor- 10 .
\end{abstract}

Key words. ISM: general - submillimeter: ISM - galaxies: ISM - ISM: clouds - Magellanic Clouds

\section{Introduction}

The radiative feedback of star formation on its progenitor molecular gas is of great interest in modern astrophysics because it can be a determining factor in the regulation of star formation in galaxies. In particular, there is special interest in the interaction of stellar far-ultraviolet (FUV) photons with a lower metallicity, lower dust-to-gas ratio gas, because it is thought to be the material from which stars formed at earlier cosmological times.

The region of the interstellar medium where the chemistry and thermal balance is dominated by the influence of stellar FUV photons is called a photon-dominated region (or photodissociation region, or PDR; Hollenbach \& Tielens 1999, and references therein). Owing to the reduced attenuation of FUV photons by dust in low-metallicity PDRs, the $\mathrm{CO}$ molecule is more readily photo-dissociated, increasing the abundance of $\mathrm{C}^{+}$and $\mathrm{C}$ relative to that of $\mathrm{CO}$. Therefore, the observational signatures of lowmetallicity gas (increased $\left[\mathrm{C} \mathrm{II}_{\mathrm{II}}\right] / \mathrm{CO}$ and $\left[\mathrm{C}_{\mathrm{I}}\right] / \mathrm{CO}$ intensity ratios) are a result of the combined effect of a strong FUV radiation field and of reduced metallicity. It is therefore difficult to study separately the influence of these two parameters on the properties of the low-metallicity molecular gas. 

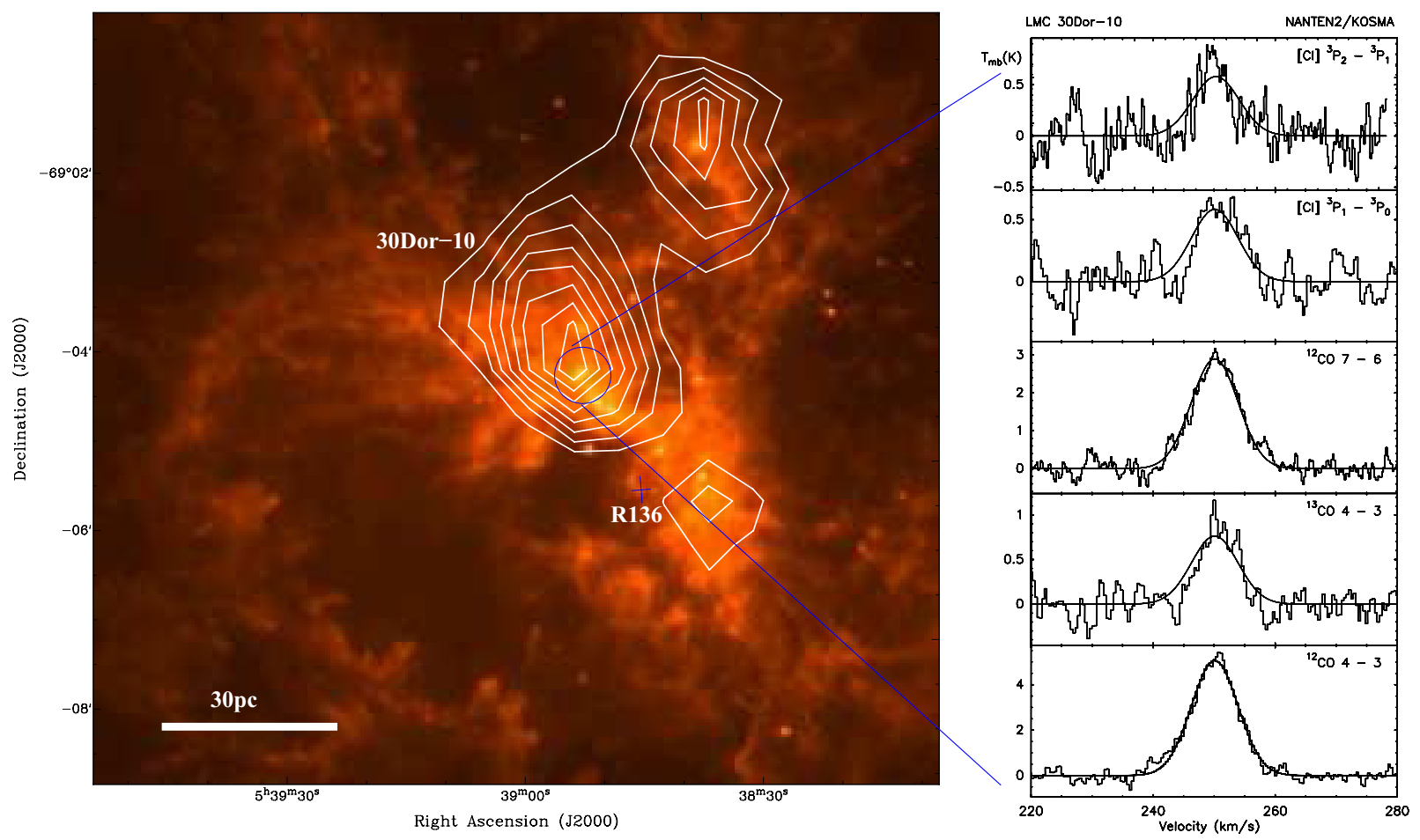

Fig. 1. (Left panel) Spitzer $8 \mu \mathrm{m}$ emission map of the 30 Dor region (Meixner et al. 2006) overlaid by an integrated intensity map of the ${ }^{12} \mathrm{CO} J=$ $1 \rightarrow 0$ line (Wong et al. 2011). The contour levels correspond to $20 \%$ to $80 \%$ of the peak intensity $\left(11.3 \mathrm{~K} \mathrm{~km} \mathrm{~s}^{-1}\right.$ ), in steps of $10 \%$. The angular resolution of the Spitzer $8 \mu \mathrm{m}$ and CO maps are 2" and 45", respectively. The center of the R136 super star cluster and the NANTEN2 38" beam are marked by a cross and a circle, respectively. (Right panel) Observed lines and corresponding Gaussian fits.

The Large Magellanic Cloud is an ideal extragalactic laboratory in which to study star formation in low-metallicity environments. Its proximity (50 kpc; Feast 1999) and nearly face-on orientation $\left(i=35^{\circ}\right.$; van der Marel \& Cioni 2001) allow us to isolate individual clouds and study them at the highest spatial resolution possible for external galaxies. The LMC also shows strongly varying FUV radiation fields and therefore very different physical conditions in a low-metallicity $\left(Z_{\odot} \simeq 0.4 Z_{\odot}\right.$; Westerlund 1997) interstellar medium.

Regions of special interest are the molecular clouds associated with the 30 Doradus H II complex. This complex is powered by one of the most dense and massive star clusters in the local universe, R136. Therefore, the 30 Doradus region exemplifies the special case in which the low-metallicity molecular gas is exposed to a very strong FUV radiation field. Among several CO-clouds located around R136, the 30 Dor-10 cloud (Johansson et al. 1998) is associated with the center of the 30 Dor nebula. The Spitzer $8 \mu \mathrm{m}$ and CO maps shown in Fig. 1 show that this region is the brightest $\mathrm{CO}$ cloud at the center of 30 Dor and is located at a projected distance of $20 \mathrm{pc}$ from the center of R136. This proximity to R136 and the presence of associated $8 \mu \mathrm{m}$ sources, which might indicate embedded star formation, suggest that the gas in 30 Dor-10 is exposed to an extreme FUV environment. The CO peak of 30 Dor-10 also associated with the [C II] (Poglitsch et al. 1995) and FIR (Werner et al. 1978) peaks. Although 30 Dor-10 is the CO peak at the center of the 30 Dor nebula, the sub-mm CO and $\left[\mathrm{C}_{\mathrm{I}}\right]$ line intensities are weaker compared with other active regions in the LMC (e.g. N159W; Pineda et al. 2008). On scales of about 10 pc, several low- $J$ transitions of ${ }^{12} \mathrm{CO}$ and ${ }^{13} \mathrm{CO}$ have been observed in this cloud (Johansson et al. 1998; Minamidani et al. 2008, 2011) as well as the [C $\left.\mathrm{C}_{\mathrm{II}}\right] 158 \mu \mathrm{m}$ line (Boreiko \& Betz 1991; Poglitsch et al. 1995). At larger spatial scales of about $50 \mathrm{pc}$, the
${ }^{12} \mathrm{CO} J=7 \rightarrow 6,{ }^{12} \mathrm{CO} J=4 \rightarrow 3$ and $\left[\mathrm{C}_{\mathrm{I}}\right]{ }^{3} \mathrm{P}_{1} \rightarrow{ }^{3} \mathrm{P}_{0}$ lines have been observed with the AST/RO telescope (Stark et al. 1997; Kim 2006).

In this paper we present observations of the ${ }^{12} \mathrm{CO} J=4 \rightarrow 3$, $J=7 \rightarrow 6$, and ${ }^{13} \mathrm{CO} J=4 \rightarrow 3$ rotational and $\left[\mathrm{C}_{\mathrm{I}}\right]{ }^{3} \mathrm{P}_{1} \rightarrow{ }^{3} \mathrm{P}_{0}$ and ${ }^{3} \mathrm{P}_{2} \rightarrow{ }^{3} \mathrm{P}_{1}$ fine-structure transitions in the 30 Dor-10 cloud in the LMC using the NANTEN2 telescope. The angular resolution of these observations allow us for the first time to study submillimeter lines at spatial scales of about $10 \mathrm{pc}$. We complement this data set with low- $J{ }^{12} \mathrm{CO}$ and ${ }^{13} \mathrm{CO}$ transitions observed with Mopra, and ASTE, with similar angular resolution.

The physical conditions of the molecular gas in 30 Dor- 10 are compared with those derived in a region with a similar metallicity but with a more moderate incident FUV radiation field, the LMC N159W region (Pineda et al. 2008). N159W is located in one the edges of the N159 H II region and is actively forming massive stars, as suggested by the presence of several embedded massive young stellar objects (Chen et al. 2010). However, the FUV and $\mathrm{H} \alpha$ fluxes in N159 are 10-20 and 7 times lower than in the 30 Dor region, respectively, suggesting that although N159W is an active region it does not have the extreme FUV environment to which 30 Dor-10 is exposed. The comparison between the physical conditions of the gas in 30 Dor- 10 and N159W will help us to understand the effects of the strength of the FUV field on the properties of the low-metallicity molecular gas in the LMC.

This paper is organized as follows. We describe the NANTEN2 observations in Sect. 2 and the observational results in Sect. 3. We derive physical properties of the low-metallicity molecular gas using a radiative transfer code independently for the $\mathrm{CO}$ and $[\mathrm{Cr}]$ lines in Sect. 4. We give a self-consistent solution of the chemistry and thermal balance of the gas using a clumpy photon-dominated region (PDR) model in Sect. 5. We 
Table 1. Gaussian-fit line parameters derived from the NANTEN2 observations of LMC 30Dor-10 and from the literature.

\begin{tabular}{|c|c|c|c|c|}
\hline Line & $\begin{array}{l}\text { Amplitude }^{a} \\
{[\mathrm{~K}]}\end{array}$ & $\begin{array}{l}\text { Center }^{b} \\
{\left[\mathrm{~km} \mathrm{~s}^{-1}\right]}\end{array}$ & $\begin{array}{c}F W H M^{b} \\
{\left[\mathrm{~km} \mathrm{~s}^{-1}\right]}\end{array}$ & $\begin{array}{c}\text { Integrated intensity } \\
{\left[\mathrm{K} \mathrm{km} \mathrm{s}^{-1}\right]}\end{array}$ \\
\hline \multicolumn{5}{|l|}{ NANTEN2 data } \\
\hline${ }^{12} \mathrm{CO} J=4 \rightarrow 3$ & $5.06(0.19)$ & $250.1(0.053)$ & $9.02(0.13)$ & $48.5(1.9)$ \\
\hline${ }^{12} \mathrm{CO} J=7 \rightarrow 6$ & $2.89(0.19)$ & & & $27.7(1.7)$ \\
\hline${ }^{13} \mathrm{CO} J=4 \rightarrow 3$ & $0.76(0.14)$ & & & $7.3(1.3)$ \\
\hline$\left[\mathrm{C}_{\mathrm{I}}\right]{ }^{3} \mathrm{P}_{1} \rightarrow{ }^{3} \mathrm{P}_{0}$ & $0.58(0.14)$ & & & $5.6(1.3)$ \\
\hline$\left[\mathrm{C}_{\mathrm{I}}\right]{ }^{3} \mathrm{P}_{2} \rightarrow{ }^{3} \mathrm{P}_{1}$ & $0.58(0.19)$ & & & $5.6(1.8)$ \\
\hline \multicolumn{5}{|l|}{ Literature datac } \\
\hline${ }^{12} \mathrm{CO} J=1 \rightarrow 0$ & $1.1(0.03)$ & $250.3(0.1)$ & $8.79(0.23)$ & $11.3(0.27)$ \\
\hline${ }^{12} \mathrm{CO} J=3 \rightarrow 2$ & $5.2(0.4)$ & $250.4(0.1)$ & $8.4(0.23)$ & $49.6(0.27)$ \\
\hline${ }^{13} \mathrm{CO} J=1 \rightarrow 0$ & $0.17(0.02)$ & $250.7(0.27)$ & $5.35(0.64)$ & $0.88(0.12)$ \\
\hline$\left[\mathrm{C}_{\mathrm{II}}\right]{ }^{2} \mathrm{P}_{3 / 2} \rightarrow{ }^{2} \mathrm{P}_{1 / 2}$ & $6.3(0.6)$ & $247.8(1.6)$ & $20.5(1.8)$ & $109.4(8.5)$ \\
\hline
\end{tabular}

Notes. ${ }^{(a)}$ The rms noise for lines observed with NANTEN2 are for channel widths of $0.37 \mathrm{~km} \mathrm{~s}^{-1}$ and $0.21 \mathrm{~km} \mathrm{~s}^{-1}$ for the $460-490$ and $810 \mathrm{GHz}$ channels, respectively. ${ }^{(b)}$ Center and width fixed to ${ }^{12} \mathrm{CO} J=4 \rightarrow 3$ best-fit values for NANTEN2 data other than this line. ${ }^{(c)}$ References for literature data are presented in Sect. 3.2.

compare our results to those constrained in the LMC-N159W region in Sect. 6.1 and we study the $\mathrm{CO}$ spectral line distribution in both regions in Sect. 6.2. We summarize the results in Sect. 7.

\section{Observations}

We used the new NANTEN2 4-m telescope situated at $4865 \mathrm{~m}$ altitude at Pampa la Bola in northern Chile to observe the ${ }^{12} \mathrm{CO} J=4 \rightarrow 3(461.0408 \mathrm{GHz}), J=7 \rightarrow 6(806.6517 \mathrm{GHz})$, and ${ }^{13} \mathrm{CO} J=4 \rightarrow 3(440.7654 \mathrm{GHz})$ rotational and [C I] ${ }^{3} \mathrm{P}_{1}-{ }^{3} \mathrm{P}_{0} \quad(492.1607 \mathrm{GHz})$ and ${ }^{3} \mathrm{P}_{2}-{ }^{3} \mathrm{P}_{1} \quad(809.3446 \mathrm{GHz})$ fine-structure transitions toward LMC 30 Dor-10. The line parameters derived from the NANTEN2 observations of LMC 30 Dor-10 are listed in Table 1 and the observed spectra are shown in Fig. 1.

The observations were made toward the peak ${ }^{12} \mathrm{CO} J=1 \rightarrow$ 0 intensity position in 30 Dor-10 (Johansson et al. 1998), located at $\alpha=5^{\mathrm{h}} 38^{\mathrm{m}} 48^{\mathrm{s}} .56$ and $\delta=-69^{\circ} 04^{\prime} 43^{\prime \prime} .2$ (J2000), using the total-power observing mode. We used a reference position at $\alpha=5^{\mathrm{h}} 39^{\mathrm{m}} 33^{\mathrm{s}} .7$ and $\delta=-69^{\circ} 04^{\prime} 00^{\prime} 0(\mathrm{~J} 2000)$, which is free of ${ }^{12} \mathrm{CO} J=1 \rightarrow 0$ emission. The duration of each beam switch cycle was varied between 20 and $30 \mathrm{~s}$, depending on the atmospheric stability during the observations. The final spectra result from total on-position integration times of $40 \mathrm{~min}$ to about $3 \mathrm{~h}$. The pointing was checked regularly on Jupiter, IRC+10216, and IRc2 in OrionA. The applied corrections were always smaller than $10^{\prime \prime}$. We do not expect significant uncertainties in the line fluxes due to pointing errors because the $\mathrm{CO}$ flux distribution in 30 Dor- 10 is smooth at scales smaller than $10^{\prime \prime}$ (e.g. Minamidani et al. 2008). The angular resolution of the observations is $38^{\prime \prime}$ for the $460-490 \mathrm{GHz}$ and $26^{\prime \prime}$ for the $810 \mathrm{GHz}$ channel, respectively. The main beam efficiencies are 0.5 and 0.4 for the $460-490 \mathrm{GHz}$ and $810 \mathrm{GHz}$ channels, respectively.

The observations were conducted with a dual-channel $460 / 810 \mathrm{GHz}$ receiver installed for verifying the submillimiter performance of the telescope. Double-sideband (DSB) receiver temperatures were $\sim 250 \mathrm{~K}$ in the lower channel and $\sim 750 \mathrm{~K}$ in the upper channel. The intermediate frequencies (IF) are $4 \mathrm{GHz}$ and $1.5 \mathrm{GHz}$, respectively. The latter IF allows simultaneous observations of the ${ }^{12} \mathrm{CO} J=7 \rightarrow 6$ line in the lower and of the $\left[\mathrm{C}_{\mathrm{I}}\right]{ }^{3} \mathrm{P}_{2}-{ }^{3} \mathrm{P}_{1}$ line in the upper sideband. These two lines were observed simultaneously with one of the lines in the
$460 \mathrm{GHz}$ channel. As backends we used two acousto-optical spectrometers (AOS) with a bandwidth of $1 \mathrm{GHz}$ and a channel resolution of $0.37 \mathrm{~km} \mathrm{~s}^{-1}$ at $460 \mathrm{GHz}$ and $0.21 \mathrm{~km} \mathrm{~s}^{-1}$ at $806 \mathrm{GHz}$.

As discussed in Pineda et al. (2008), the main sources of uncertainty in the absolute line calibration are the precision with which the beam efficiencies are determined and the accuracy of the atmospheric calibration. We estimate that both sources of uncertainty contribute to a total absolute calibration uncertainty of $20 \%$. In our analysis we added these errors quadratically with the radiometric noise, assumed to be given by the formal errors of the Gaussian fits. Propagating these absolute calibration uncertainties for line ratios between lines in the 460-490 and $810 \mathrm{GHz}$ channels, we obtain a relative calibration uncertainty of $28 \%$. Line ratios between lines located in the same frequency channel do not suffer from the uncertainty on the determination of the beam efficiencies $(\sim 10 \%)$ and we propagate the errors only considering the uncertainties on the atmospheric calibration $(\sim 17 \%)$.

\section{Observational results}

\subsection{NANTEN2 data}

The spectra observed toward 30 Dor-10 are shown in Fig. 1, together with Gaussian fits whose results are listed in Table 1. We determined the line center and width from the high signal-tonoise ${ }^{12} \mathrm{CO} J=4 \rightarrow 3$ spectrum and we held them fixed for the Gaussian fit of the line amplitude of the other lines. This method is adequate because their profiles are, within their limited signalto-noise ratio, consistent with that of the ${ }^{12} \mathrm{CO} J=4 \rightarrow 3$ line.

To calculate intensity ratios between lines in the upper and lower frequency channels we need to account for the different beam sizes (38" and 26", respectively). We therefore determined the source extent using lower resolution data. As described in Pineda et al. (2008), assuming a Gaussian source distribution with FWHM $\Theta_{\mathrm{s}}$ and a source peak brightness temperature of $T_{\mathrm{s} \text {,peak }}$, the beam filling correction gives a main beam brightness temperature in a beam of $F W H M \Theta_{\mathrm{b}}$ of $T_{\mathrm{mb}}=\Theta_{\mathrm{s}}^{2} /\left(\Theta_{\mathrm{s}}^{2}+\Theta_{\mathrm{b}}^{2}\right) T_{\mathrm{s} \text {,peak. }}$. The ratio of the main beam brightness temperature in two different beams 1 and 2 is given by $R_{1,2}=T_{\mathrm{mb}, 1} / T_{\mathrm{mb}, 2}=\left(\Theta_{\mathrm{b}, 2}^{2}+\Theta_{\mathrm{s}}^{2}\right) /\left(\Theta_{\mathrm{b}, 1}^{2}+\Theta_{\mathrm{s}}^{2}\right)$. With this, we can 
A\&A 544, A84 (2012)

Table 2. Observed line intensity ratios, corrected for beam coupling to an assumed common FWHM source size of 90".

\begin{tabular}{lcccc}
\hline \hline Species & Ratio $^{a}$ & Fit error & Calibration error & Total error \\
\hline${ }^{12} \mathrm{CO} J=7 \rightarrow 6 /{ }^{12} \mathrm{CO} J=4 \rightarrow 3$ & 0.52 & 0.04 & $28 \%$ & 0.15 \\
{$\left[\mathrm{C}_{\mathrm{I}}\right]{ }^{3} \mathrm{P}_{2} \rightarrow{ }^{3} \mathrm{P}_{1} /[\mathrm{CI}]{ }^{3} \mathrm{P}_{1} \rightarrow{ }^{3} \mathrm{P}_{0}$} & 0.92 & 0.37 & $28 \%$ & 0.45 \\
${ }^{13} \mathrm{CO} J=4 \rightarrow 3 /{ }^{12} \mathrm{CO} J=4 \rightarrow 3$ & 0.15 & 0.03 & $17 \%$ & 0.05 \\
{$\left[\mathrm{C}_{\mathrm{I}}\right]{ }^{3} \mathrm{P}_{2} \rightarrow{ }^{3} \mathrm{P}_{1} / \mathrm{CO} J=7 \rightarrow 6$} & 0.20 & 0.07 & 17 & 0.09 \\
${ }^{12} \mathrm{CO} J=1 \rightarrow 0 /{ }^{12} \mathrm{CO} J=4 \rightarrow 3$ & 0.22 & 0.05 & $28 \%$ & 0.08 \\
${ }^{13} \mathrm{CO} J=1 \rightarrow 0 /{ }^{12} \mathrm{CO} J=1 \rightarrow 0$ & 0.08 & 0.01 & $28 \%$ & 0.02 \\
{$\left[\mathrm{C}_{\mathrm{II}}\right]{ }^{2} \mathrm{P}_{3 / 2} \rightarrow{ }^{2} \mathrm{P}_{1 / 2} /{ }^{12} \mathrm{CO} J=4 \rightarrow 3$} & 1.24 & 0.13 & $28 \%$ & 0.37 \\
\hline
\end{tabular}

Notes. ${ }^{(a)}$ Ratio of amplitudes from Gaussian fits.

derive the source size from the observed brightness ratio in two beam sizes:

$\Theta_{\mathrm{s}}^{2}=\frac{R_{1,2} \Theta_{\mathrm{b}, 1}^{2}-\Theta_{\mathrm{b}, 2}^{2}}{1-R_{1,2}}$.

With the source size we can correct the observed intensity ratios for the beam coupling of the different beam sizes at two observing bands $x$ and $y$ to the source intrinsic intensity ratio $R_{\mathrm{S}}$ via

$R_{\mathrm{s}}=\frac{T_{\mathrm{mb}, x}}{T_{\mathrm{mb}, y}} \frac{\Theta_{\mathrm{s}}^{2}+\Theta_{\mathrm{b}, x}^{2}}{\Theta_{\mathrm{s}}^{2}+\Theta_{\mathrm{b}, y}^{2}}$

We used observations of the $\left[\mathrm{C}_{\mathrm{I}}\right]{ }^{3} \mathrm{P}_{1} \rightarrow{ }^{3} \mathrm{P}_{0}$ line made with the SWAS satellite (Frank Bensch, priv. comm.) with an angular resolution of 258". The integrated line intensity of this line observed by SWAS is $0.44 \mathrm{~K} \mathrm{~km} \mathrm{~s}^{-1}$. With NANTEN2 we obtained an integrated line intensity of $5.58 \mathrm{~K} \mathrm{~km} \mathrm{~s}^{-1}$ in a $38^{\prime \prime}$ beam. Thus, from Eq. (1) we obtain a source size of $\Theta_{\mathrm{s}}=64^{\prime \prime}$, which according to Eq. (2), corresponds to a $18 \%$ correction to the line ratios between the upper and lower channels. We also determined the source size by considering the ${ }^{12} \mathrm{CO} J=1 \rightarrow 0$ integrated line intensity observed by the NANTEN telescope (e.g. Fukui et al. 2008) of $4.1 \mathrm{~K} \mathrm{~km} \mathrm{~s}^{-1}$ in a $158^{\prime \prime}$ beam and by the Mopra telescope (Jürgen Ott, priv. comm.) of $11.3 \mathrm{~K} \mathrm{~km} \mathrm{~s}^{-1}$ in a 33" beam. Equation (1) results in a source size of $\Theta_{\mathrm{s}}=110^{\prime \prime}$, corresponding to a $8 \%$ correction. We assumed an intermediate value for the source size of $\Theta_{\mathrm{s}}=90^{\prime \prime}$ corresponding to a correction of $10 \%$. A source size of $90^{\prime \prime}$ is consistent with the angular size of 30 Dor-10's CO-emitting region shown in Fig. 1. We note that assuming a source size introduces an uncertainty of about $5 \%$ to the line ratios between the upper and lower channels. The correction also assumes that the source structure is the same at the spatial scales observed with the 460 and $810 \mathrm{GHz}$ channels. The validity of this assumption likely dominates the uncertainties in the line ratios. However, as we will see in Sects. 4 and 5, the good fit obtained for the physical parameters supports this assumption. Table 2 shows the line ratios thus corrected, which we used for the excitation analysis discussed below.

Kim et al. (2005) presented maps of the ${ }^{12} \mathrm{CO} J=4 \rightarrow 3$ line in the 30 Dor region with a $109^{\prime \prime}$ beam using the AST/RO telescope. In addition to the ${ }^{12} \mathrm{CO} J=4 \rightarrow 3$ component at $\sim 250 \mathrm{~km} \mathrm{~s}^{-1}$ they detected another component at $\sim 302 \mathrm{~km} \mathrm{~s}^{-1}$, a factor of $\sim 3$ weaker. We did not find this additional component in our ${ }^{12} \mathrm{CO} J=4 \rightarrow 3$ spectrum. Considering their derived FWHM line-width for this component of $5.1 \mathrm{~km} \mathrm{~s}^{-1}$, we derive an $3 \sigma$ upper limit of $0.52 \mathrm{~K} \mathrm{~km} \mathrm{~s}^{-1}$, which is a factor of $\sim 10$ weaker than the main component of ${ }^{12} \mathrm{CO} J=4 \rightarrow 3$ in 30 Dor- 10 .

\subsection{Additional lines from the literature}

To better constrain the physical parameters of the emitting gas in 30 Dor-10, we combined the lines observed with NANTEN2 with additional observations of the ${ }^{12} \mathrm{CO} J=1 \rightarrow 0, J=3 \rightarrow 2$, ${ }^{13} \mathrm{CO} J=1 \rightarrow 0$ and $\left[\mathrm{C}_{\mathrm{II}}\right] 158 \mu \mathrm{m}$ lines. The ${ }^{12} \mathrm{CO}$ and ${ }^{13} \mathrm{CO} J=1 \rightarrow 0$ lines were observed with the ATNF Mopra $22 \mathrm{~m}$ telescope (Jürgen Ott, priv. comm.) at the position of the NANTEN2 observations. The data have an angular resolution of $33^{\prime \prime}$ and a channel width of $0.7 \mathrm{~km} \mathrm{~s}^{-1}$. The ${ }^{12} \mathrm{CO} J=3 \rightarrow 2$ observations were observed with the ASTE telescope and were presented by Minamidani et al. (2008). The angular resolution for both lines is about $22^{\prime \prime}$ and the channel width is $0.45 \mathrm{~km} \mathrm{~s}^{-1}$. The Kuiper Airborne Observatory (KAO) observed velocityresolved [C $\mathrm{II}] 158 \mu \mathrm{m}$ emission toward 30 Dor-10 with an angular resolution of 43" (Boreiko \& Betz 1991). The pointing of the KAO observations is about $20^{\prime \prime}$ farther southwest than the NANTEN2 position. We adopted their values without further corrections. Indeed, the integrated intensity of the $\left[\mathrm{C}_{\mathrm{II}}\right]$ line from the KAO observations of $7.7 \times 10^{-4} \mathrm{erg} \mathrm{cm}^{-2} \mathrm{~s}^{-1} \mathrm{sr}^{-1}$ is consistent to within $30 \%$ with the integrated intensity at the position of the NANTEN2 observations in the [C II] map published by Poglitsch et al. (1995), observed with low-velocity resolution $\left(\Delta v=75 \mathrm{~km} \mathrm{~s}^{-1}\right)$ using the FIFI spectral imaging instrument on the KAO. In Table 3 we present a summary of rest frequencies, energies above the ground level, and critical densities of the spectral lines used in our analysis.

We assumed that the uncertainty of the absolute calibration of all independently observed lines is about $20 \%$, and propagated these errors to obtain a line ratio calibration accuracy of $28 \%$. Table 2 quotes the derived total errors, obtained by quadratically summing the formal fit error and the calibration uncertainty.

\section{Excitation analysis}

We first constrained the properties of the emitting gas by comparing line intensity ratios and absolute intensities with the results of a radiative transfer code. We analyzed [ $\left.\mathrm{C}_{\mathrm{I}}\right]$ and $\mathrm{CO}$ separately, but assume that they originate from the same regions with identical beam filling factors. This assumption is justified considering that the $\mathrm{CO}$ and $\left[\mathrm{C}_{\mathrm{I}}\right]$ emission arise from the FUV illuminated surfaces of clouds that are unresolved at the scale of our observations.

We used the RADEX non-LTE radiative transfer code by van der Tak et al. (2007), using the uniform sphere approximation, to calculate line intensities as a function of the kinetic temperature, $\mathrm{H}_{2}$ volume density, and the column density per velocity interval $N / \Delta v$ of the species of interest. The collision cross sections were taken from the Leiden Atomic and Molecular Database (LAMDA; Schöier et al. 2005). The code provides the average line intensity of a spherical cloud. We therefore scaled 
the data by a filling factor to compare them with the observed absolute intensities. As mentioned above, considering a medium consisting of clumps that are much smaller than the scale of our observations, the $\mathrm{CI}$ and $\mathrm{CO}$ species can be considered as occupying the same volume, hence the line ratios are independent of beam dilution. For optically thin clumps (or for clumps with moderate opacity), the observed-to-model integrated intensity ratio gives the ratio of the beam-averaged column density to the clump intrinsic column density, assuming that the total integrated intensity is proportional to the number of clumps, i.e. no velocity crowding is present. We therefore determined the beam filling by comparing the observed absolute intensity of a line with a constrained model.

\section{1. [C I]-emission}

In Table 2 we see that the ratio between the two $\left[\mathrm{C}_{\mathrm{I}}\right]$ lines is 0.92 . We compare this ratio with predictions from the radiative transfer calculations in Fig. 2. We present model line ratios for three different kinetic temperatures 80, 120, and $160 \mathrm{~K}$, as a function of the $\left[\mathrm{C}_{\mathrm{I}}\right]$ column density per velocity interval, $N_{\text {[C I] }} / \Delta v$, and $\mathrm{H}_{2}$ volume density, $n\left(\mathrm{H}_{2}\right)$. The coverage in $N_{\text {[C I] }} / \Delta v$ is $10^{15}-10^{19} \mathrm{~cm}^{-2}$ and in $n\left(\mathrm{H}_{2}\right)$ is $10^{2}-10^{6} \mathrm{~cm}^{-3}$. The observed ratio can only be reproduced by kinetic temperatures above $60 \mathrm{~K}$ and $\mathrm{H}_{2}$ densities of about $1-3 \times 10^{3} \mathrm{~cm}^{-3}$. This $\mathrm{H}_{2}$ density is similar to, or somewhat higher than, the critical density of the $\left[\mathrm{C}_{\mathrm{I}}\right]{ }^{3} \mathrm{P}_{1} \rightarrow{ }^{3} \mathrm{P}_{0}$ line $\left(\sim 1 \times 10^{3} \mathrm{~cm}^{-3}\right)$, and therefore its emission is close to being thermalized. From the analysis of the $\mathrm{CO}$ emission (see below) we found that the beamfilling factor in 30 Dor- 10 is about $1 / 16$, so that the observed $\left[\mathrm{C}_{\mathrm{I}}\right]{ }^{3} \mathrm{P}_{1}-{ }^{3} \mathrm{P}_{0}$ brightness of $0.58 \mathrm{~K}$ should be scaled up to $9.3 \mathrm{~K}$. This brightness temperature corresponds to a $\left[\mathrm{C}_{\mathrm{I}}\right]$ column density per velocity interval of about $1 \times 10^{17} \mathrm{~cm}^{-2} / \mathrm{km} \mathrm{s}^{-1}$. The multiplication by a filling factor is appropriate because, in the range of kinetic temperatures and $\mathrm{H}_{2}$ densities inferred above, the $\left[\mathrm{C}_{\mathrm{I}}\right]{ }^{3} \mathrm{P}_{1}-{ }^{3} \mathrm{P}_{0}$ line is likely optically thin.

\subsection{CO-emission}

We compare the observed ${ }^{12} \mathrm{CO} J=7 \rightarrow 6 /{ }^{12} \mathrm{CO} J=4 \rightarrow 3$ and ${ }^{13} \mathrm{CO} J=4 \rightarrow 3 /{ }^{12} \mathrm{CO} J=4 \rightarrow 3$ ratios with predictions of the radiative transfer code in Fig. 3. We assumed the ${ }^{12} \mathrm{CO} /{ }^{13} \mathrm{CO}$ abundance ratio of 35 derived in 30 Dor- 10 by Heikkilä et al. (1999). We required that the volume density of the CO-emitting region is equal to, or somewhat higher than, that constrained for the $\left[\mathrm{C}_{\mathrm{I}}\right]$ emission, because we expect that both [C $\mathrm{I}]$ and mid-J CO lines are emitted from adjacent layers in a PDR-like structure ${ }^{1}$, where the neutral carbon is located in an equal or lower-density region compared to the one at which $\mathrm{CO}$ is located, and that the cloud's volume density profile is smooth. In Fig. 3, this condition is only valid for kinetic temperatures higher than $120 \mathrm{~K}$ for a volume density range between $3 \times 10^{3}-10^{4} \mathrm{~cm}^{-3}$. The intersection of the two $\mathrm{CO}$ line ratios constrains the $\mathrm{CO}$ column density per velocity interval to about $3 \times 10^{17} \mathrm{~cm}^{-2} / \mathrm{km} \mathrm{s}^{-1}$ at $T_{\text {kin }} \simeq 160 \mathrm{~K}$. The derived kinetic temperature and $\mathrm{H}_{2}$ density are consistent with previously published excitation analyzes based on CO observations (Johansson et al. 1998; Kim 2006; Minamidani et al. 2008, 2011).

Figure 4 shows that the model-predicted clump-averaged absolute line intensity of the ${ }^{13} \mathrm{CO} J=4 \rightarrow 3$ line, at the column

\footnotetext{
${ }^{1}$ For an example of a typical temperature distribution and abundance structure of the main carbon species in PDRs, see e.g. Figs. 7 and 9 in Tielens \& Hollenbach (1985).
}
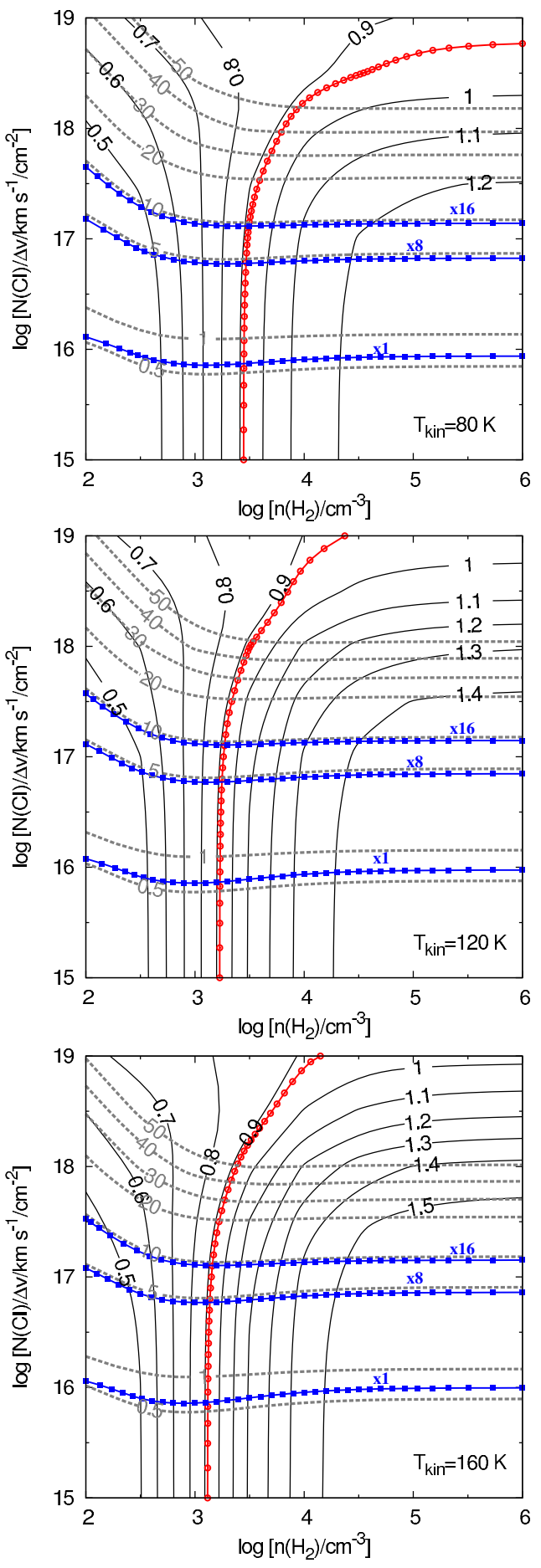

Fig. 2. Comparison between the observations and the predictions from the radiative transfer model for the $\left[\mathrm{C}_{\mathrm{I}}\right]{ }^{3} \mathrm{P}_{2} \rightarrow{ }^{3} \mathrm{P}_{1} /[\mathrm{C} \mathrm{I}]{ }^{3} \mathrm{P}_{1} \rightarrow{ }^{3} \mathrm{P}_{0}$ line ratio (black-solid lines) and the $\left[\mathrm{C}_{\mathrm{I}}{ }^{3} \mathrm{P}_{1} \rightarrow{ }^{3} \mathrm{P}_{0}\right.$ absolute intensity (graydashed lines) for kinetic temperatures of 80,120 , and $160 \mathrm{~K}$. The observed $\left[\mathrm{C}_{\mathrm{I}}\right]{ }^{3} \mathrm{P}_{2} \rightarrow{ }^{3} \mathrm{P}_{1} /\left[\mathrm{C}_{\mathrm{I}}\right]{ }^{3} \mathrm{P}_{1} \rightarrow{ }^{3} \mathrm{P}_{0}$ line ratio of 0.92 is shown as a red line with circles. The observed $\left[\mathrm{C}_{\mathrm{I}}\right]{ }^{3} \mathrm{P}_{1} \rightarrow{ }^{3} \mathrm{P}_{0}$ line intensity of $0.58 \mathrm{~K}$ and its value scaled by a beam filling factor of $1 / 8$ and $1 / 16$ are shown as blue lines with boxes.

and volume density regime constrained by the observed line ratios, is about 16 times higher than that observed at $T_{\text {kin }}=160 \mathrm{~K}$. This implies a beam filling factor for $\mathrm{CO}$ of about $1 / 16$, which corresponds to a typical clump size of $10^{\prime \prime}$. Recent ATCA observations of $\mathrm{HCO}^{+}$revealed several compact clumps in 30 Dor-10 (Anderson et al., in prep.). The clumps have a typical angular 

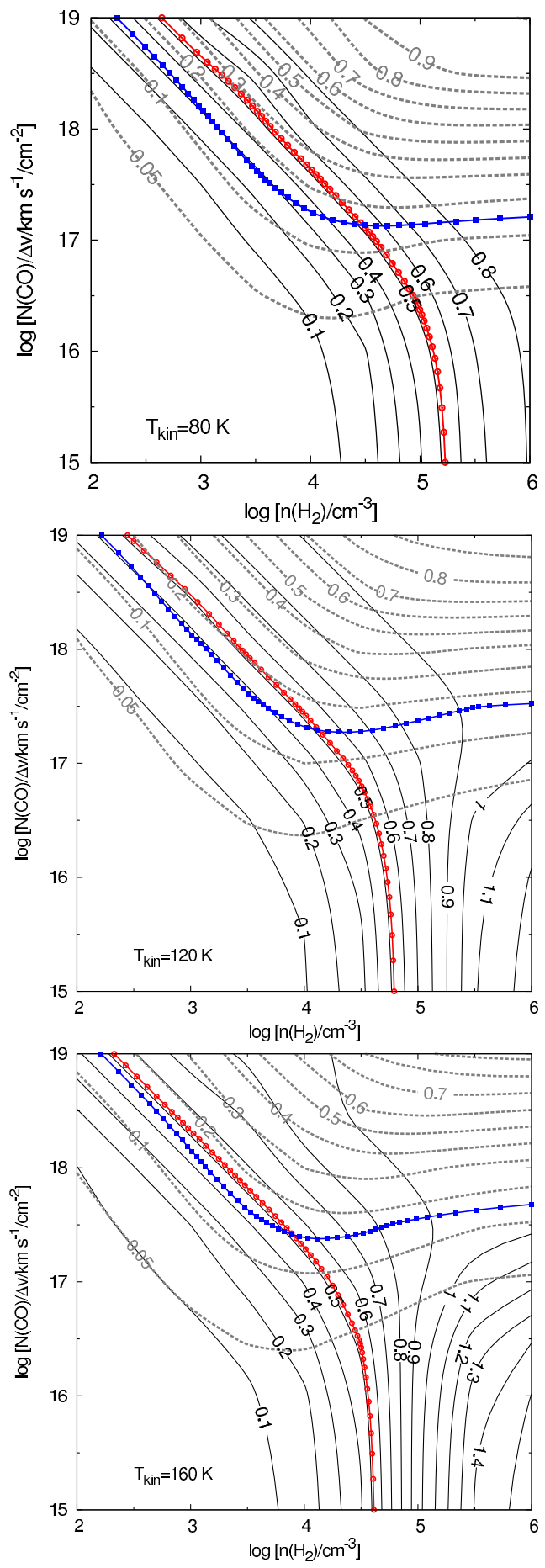

Fig. 3. Comparison between the observations and the predictions from the radiative transfer model for the ${ }^{12} \mathrm{CO} J=7 \rightarrow 6$ to $J=4 \rightarrow$ 3 (black-solid contours) and ${ }^{13} \mathrm{CO}$ to ${ }^{12} \mathrm{CO} J=4 \rightarrow 3$ (gray-dashed contours) line ratios for kinetic temperatures of 80,120 , and $160 \mathrm{~K}$. The plots assume a fractional abundance of ${ }^{12} \mathrm{CO} /{ }^{13} \mathrm{CO}$ of 35 . The observed ${ }^{12} \mathrm{CO} J=7 \rightarrow 6$ to $J=4 \rightarrow 3$ ratio of 0.52 is shown as a blue line with boxes while the ${ }^{13} \mathrm{CO}$ to ${ }^{12} \mathrm{CO} J=4 \rightarrow 3$ ratio of 0.15 is shown as a red line with circles.

size of $10^{\prime \prime}$, and are marginally resolved at the resolution of their observations, $6 "$. More observations with higher angular resolution are required to confirm the observed clump sizes in 30 Dor- 1.

In Fig. 5 we consider line ratios involving the ${ }^{12} \mathrm{CO}$ and ${ }^{13} \mathrm{CO} J=1 \rightarrow 0$ transitions for $T_{\text {kin }}=160 \mathrm{~K}$. The ${ }^{12} \mathrm{CO} J=1 \rightarrow 0$

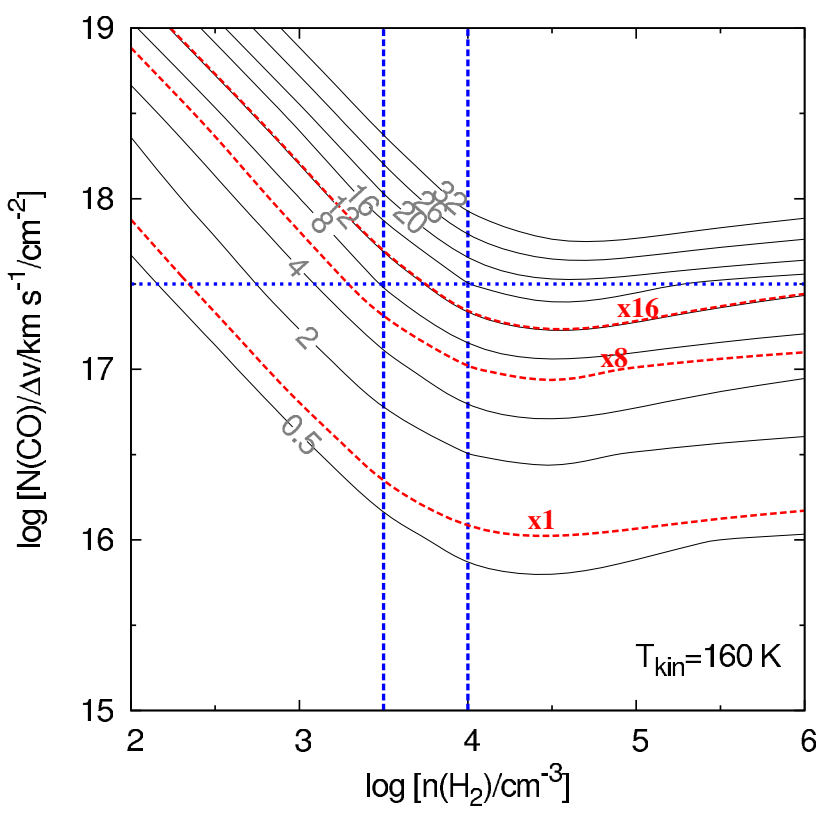

Fig. 4. Radiative transfer predictions and the observed absolute intensity of ${ }^{13} \mathrm{CO} J=4 \rightarrow 3$ for $T_{\text {kin }}=160 \mathrm{~K}$. The three red-dashed contours show the observed intensity and its value scaled up by factors of 8 and 16, respectively. The blue-dashed lines show the range of column and volume densities constrained by the analysis of the $\mathrm{CO}$ line ratios. The absolute intensity of ${ }^{13} \mathrm{CO} J=4 \rightarrow 3$ needs to be scaled up by a factor of about 16 to reproduce the constrained column and volume densities.

to $J=4 \rightarrow 3$ ratio of 0.21 , however, suggests lower column and higher volume densities than those constrained above. The low ${ }^{12} \mathrm{CO} J=1 \rightarrow 0$ to $J=4 \rightarrow 3$ ratio is due to the observed ${ }^{12} \mathrm{CO} J=1 \rightarrow 0$ line that is too weak for the physical conditions we derived. The observed ${ }^{13} \mathrm{CO} /{ }^{12} \mathrm{CO}$ ratio is therefore also affected by this weaker ${ }^{12} \mathrm{CO} J=1 \rightarrow 0$ emission. This may indicate an additional colder gas component. We were only able to reproduce absolute intensities of the ${ }^{12} \mathrm{CO}$ and ${ }^{13} \mathrm{CO} J=1 \rightarrow 0$ lines using a model with the conditions obtained for the column and volume density derived for [ $\left.\mathrm{C}_{\mathrm{I}}\right]$ (Sect. 4.1) and kinetic temperatures of $T_{\text {kin }}=10-30 \mathrm{~K}$. This is consistent with observations of higher density tracers, suggesting a colder $(\sim 50 \mathrm{~K})$ and denser $\left(\sim 10^{5} \mathrm{~cm}^{-3}\right)$ gas component in 30 Dor-10 (Heikkilä et al. 1999).

\section{3. $\left[\mathrm{CI}_{\mathrm{I}}\right]$ and $\mathrm{CO}$ column densities}

Combining our estimate of the $\mathrm{CO}$ and $\mathrm{C}_{\mathrm{I}}$ column density per velocity interval, the observed line width of $9 \mathrm{~km} \mathrm{~s}^{-1}$, and the beam filling factor of $1 / 16$, we derive a beam-averaged column density of $1.7 \times 10^{17} \mathrm{~cm}^{-2}$ and $5.6 \times 10^{16} \mathrm{~cm}^{-2}$ for $\mathrm{CO}$ and $\mathrm{C}_{\mathrm{I}}$, respectively. Thus, the ratio of the column density of $\left[\mathrm{C}_{\mathrm{I}}\right]$ to that of $\mathrm{CO}$ is $\sim 3$. Since the ${ }^{12} \mathrm{CO}$ and ${ }^{13} \mathrm{CO} J=1 \rightarrow 0$ lines indicate an additional, cooler component, this ratio is an upper limit. Assuming that all gas-phase carbon is in the form of $\mathrm{C}_{\mathrm{I}}$ and $\mathrm{CO}$ in their respective line-emitting regions, we used a carbon abundance relative to $\mathrm{H}_{2}$ in the LMC of $1.6 \times 10^{-4}$ (derived from $[\mathrm{C}] /[\mathrm{H}]=8 \times 10^{-5}$; Dufour et al. 1982) to obtain an $\mathrm{H}_{2}$ mass of $1625 M_{\odot}$ associated with $\mathrm{CO}$ and of $531 M_{\odot}$ associated

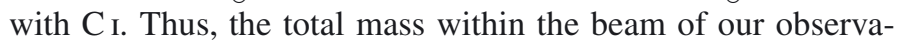
tions is $2156 M_{\odot}$. Note that our beam size of $38^{\prime \prime}$ FWHM corresponds to $9.5 \mathrm{pc}$ at the distance of the LMC and hence covers $1 / 2$ of the 30 Dor- 10 cloud's extent of about $20 \mathrm{pc}$. If the $\mathrm{H}_{2}$ column 


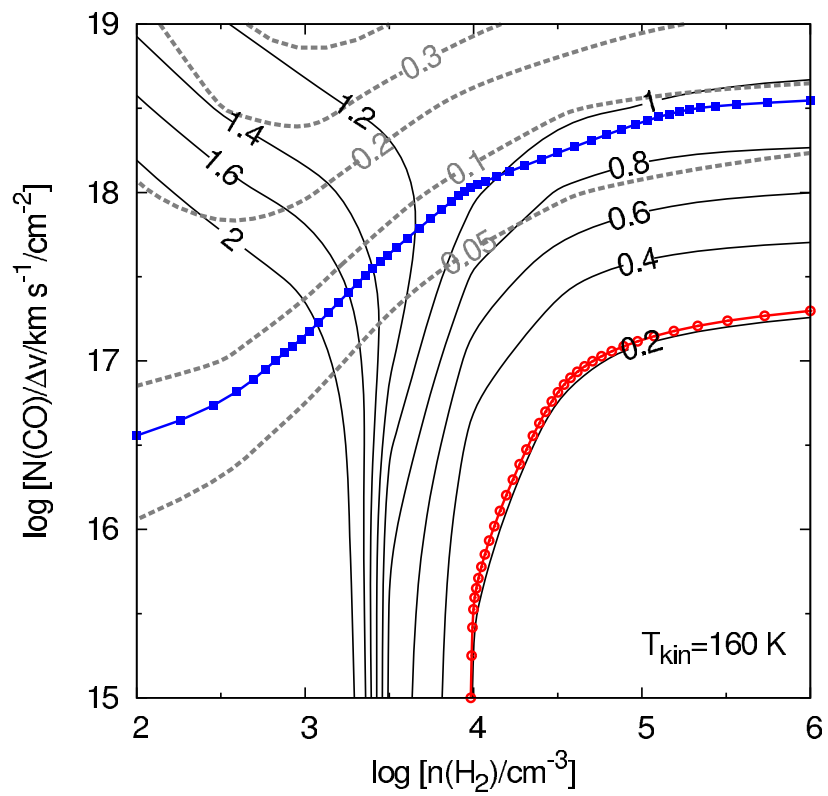

Fig. 5. Comparison between the observations and the predictions from the radiative transfer model for the ${ }^{12} \mathrm{CO} J=1 \rightarrow 0$ and $J=4 \rightarrow 3$ line ratios (black-solid contour) and the ${ }^{13} \mathrm{CO}$ to ${ }^{12} \mathrm{CO} J=1 \rightarrow 0$ line ratio (gray-dashed contour) for $T_{\text {kin }}=160 \mathrm{~K}$. The observed ${ }^{13} \mathrm{CO}$ to ${ }^{12} \mathrm{CO} J=1 \rightarrow 0$ ratio of 0.08 is shown as a blue line with boxes while the ${ }^{12} \mathrm{CO} J=1 \rightarrow 0$ to $J=4 \rightarrow 3$ ratio of 0.22 is shown as a red line with circles.

density is uniform over 30 Dor-10, the total mass of the cloud should be a factor of 4 larger than the mass derived within the beam of our observations, reflecting the difference in area. Thus, the total $\mathrm{H}_{2}$ mass in 30 Dor- 10 should be about $8.6 \times 10^{4} M_{\odot}$. This derived mass agrees well with the CO-luminosity mass of $8.5 \times 10^{4} M_{\odot}$ derived by Johansson et al. (1998) assuming a CO-to- $\mathrm{H}_{2}$ conversion factor of $4 \times 10^{20} \mathrm{~cm}^{-2}\left(\mathrm{~K} \mathrm{~km} \mathrm{~s}^{-1}\right)^{-1}$ (e.g. Israel 1997; Hughes et al. 2010; Pineda et al. 2009). Note that some $\mathrm{H}_{2}$ might be not traced by $\mathrm{CO}$ or [ $\left.\mathrm{C}_{\mathrm{I}}\right]$ but by [C $\mathrm{CI}$ ] (the "hidden $\mathrm{H}_{2}$ gas"). This can account for about 30-50\% of the $\mathrm{H}_{2}$ in the Galaxy (e.g Grenier et al. 2005; Langer et al. 2010; Velusamy et al. 2010; Planck Collaboration 2011), and is expected to have an even larger mass in low-metallicity environments (Madden et al. 1997; Wolfire et al. 2010). Therefore, the mass estimate derived here should be considered a lower limit to the total $\mathrm{H}_{2}$ mass.

\section{PDR-model analysis}

In the following we investigate whether our observations toward 30 Dor-10 can be explained in terms of an ensemble of PDRs distributed in a clumpy interstellar medium. We used the KOSMA- $\tau$ PDR model (Störzer et al. 1996; Röllig et al. 2006) to model an ensemble of spherical clumps with a power-law mass spectrum $\mathrm{d} N / \mathrm{d} M \propto M^{-1.8}$ and a mass-size relation $M \propto r^{2.3}$ (Cubick et al. 2008). The model provides a self-consistent solution of the chemistry and thermal balance. The free parameters of the clumpy PDR ensemble are (1) average ensemble total $\mathrm{H}$ volume density $\left(n_{\mathrm{H}}+2 n_{\mathrm{H}_{2}}\right), n_{\mathrm{ens}}$, (2) ensemble mass, $M_{\mathrm{ens}}$, (3) strength of the FUV radiation field in units of the Draine (1978) field, $\chi_{0}$, and (4) the minimum and maximum mass of the clump ensemble, $\left[M_{\min }, M_{\max }\right]$. We fix the metallicity in the model to $Z_{\odot}=0.4$. We attempted to fit the observed lines with a model with $Z_{\odot}=1$, but this solar metallicity model could not reproduce the mid- $J \mathrm{CO}$ observations with a $\chi^{2}$ about factor of
Table 3. Parameters for different relevant molecular and atomic transitions.

\begin{tabular}{lccc}
\hline \hline Line & Frequency $/ \mathrm{GHz}$ & $E_{\text {upper }} / \mathrm{K}$ & $n_{\text {cr }} / \mathrm{cm}^{-3}$ \\
\hline${ }^{12} \mathrm{CO} J=1 \rightarrow 0$ & 115.2712 & 5.5 & $2 \times 10^{3}$ \\
${ }^{12} \mathrm{CO} J=3 \rightarrow 2$ & 345.7959 & 33.2 & $4 \times 10^{4}$ \\
${ }^{12} \mathrm{CO} J=4 \rightarrow 3$ & 461.0408 & 55.3 & $9 \times 10^{4}$ \\
${ }^{12} \mathrm{CO} J=7 \rightarrow 6$ & 806.6517 & 154.9 & $4 \times 10^{5}$ \\
${ }^{12} \mathrm{CO} J=9 \rightarrow 8$ & 1036.912 & 248.9 & $1 \times 10^{6}$ \\
${ }^{13} \mathrm{CO} J=1 \rightarrow 0$ & 110.2013 & 5.3 & $2 \times 10^{3}$ \\
${ }^{13} \mathrm{CO} J=4 \rightarrow 3$ & 440.7654 & 52.9 & $8 \times 10^{4}$ \\
{$[\mathrm{C} \mathrm{I}]{ }^{3} \mathrm{P}_{1} \rightarrow{ }^{3} \mathrm{P}_{0}$} & 492.1607 & 23.6 & $1 \times 10^{3}$ \\
{$[\mathrm{C}]{ }^{3} \mathrm{P}_{2} \rightarrow{ }^{3} \mathrm{P}_{1}$} & 809.3446 & 62.5 & $1 \times 10^{3}$ \\
{$\left[\mathrm{O}_{\mathrm{I}}\right]{ }^{3} \mathrm{P}_{1} \rightarrow{ }^{3} \mathrm{P}_{2}$} & 4744.777 & 227.7 & $6 \times 10^{5}$ \\
{$\left[\mathrm{O}_{\mathrm{I}}\right]{ }^{3} \mathrm{P}_{0} \rightarrow{ }^{3} \mathrm{P}_{1}$} & 2060.069 & 326.6 & $7 \times 10^{4}$ \\
{$[\mathrm{C}$ II $]{ }^{2} \mathrm{P}_{3 / 2} \rightarrow{ }^{2} \mathrm{P}_{1 / 2}$} & 1900.5377 & 91.2 & $5 \times 10^{3}$ \\
\hline
\end{tabular}

Notes. The listed critical densities were calculated for collisions with $p-\mathrm{H}_{2}$ and a kinetic temperature of $100 \mathrm{~K}$. The critical densities were calculated using the Einstein A - and collisional rate coefficients taken from the Leiden Atomic and Molecular Database (LAMDA; Schöier et al. 2005).

10 larger than for the low-metallicity model. Note that we assumed that the clumps do not spatially overlap, therefore we considered optical depth effects only within individual clumps. We also assumed that the FUV field is uniform within the beam of the observations.

We constrained the model with the absolute intensities of all five lines observed with NANTEN2 together with the ${ }^{12} \mathrm{CO} J=$ $3 \rightarrow 2$ and $[\mathrm{C}$ II] $158 \mu \mathrm{m}$ lines taken from the literature (Sect. 3.2). Fitting absolute line intensities instead of line ratios allowed us to study the internal structure of the cloud (clumpiness) via the area and volume filling factors, and it adds the cloud mass and size as model parameters. The source size was fixed to be 90" (Sect. 3.1). We did not include the ${ }^{12} \mathrm{CO}$ and ${ }^{13} \mathrm{CO} J=1 \rightarrow 0$ transitions in the model fit because these transitions are likely affected by optical depth effects between clumps that are not accounted for by the model. We still compared the intensities of the ${ }^{12} \mathrm{CO}$ and ${ }^{13} \mathrm{CO} J=1 \rightarrow 0$ transitions with the predictions of the PDR model and discuss below the possible reason of the discrepancy between model and observations. We used simulated annealing to find the optimum model parameter combination that predicts absolute line intensities that match those observed.

The best-fit model results suggest an average ensemble total $\mathrm{H}$ volume density $n_{\text {ens }}=3.3 \times 10^{5} \mathrm{~cm}^{-3}$, an ensemble mass $M_{\mathrm{ens}}=1.2 \times 10^{5} M_{\odot}$. The minimum and maximum masses of the clump ensemble are $M_{\min }=1 \times 10^{-3} M_{\odot}$ and $M_{\text {max }}=0.1 M_{\odot}$. Note that $n_{\text {ens }}$ corresponds to the average total $\mathrm{H}$ volume density (i.e. $n_{\mathrm{H}}+2 n_{\mathrm{H}_{2}}$ ). Ignoring the contribution from atomic $\mathrm{H}$, the average ensemble $\mathrm{H}_{2}$ volume density is about $6 \times 10^{4} \mathrm{~cm}^{-3}$, consistent with that derived with the excitation analysis in Sect. 4. The ensemble mass is also consistent with that derived for the whole extent of the 30 Dor-10 cloud in Sect. 4.3. A comparison between the observations, the predictions from the PDR model, and the results of the excitation analysis (Sect. 4) is listed in Table 4.

The area filling factor of the clump ensemble is defined as the sum of all clump projected areas over the cloud solid angle (assumed to be $\left.90^{\prime \prime}\right)$. Similarly, the volume filling factor is defined as the sum of all clump volumes over the volume of a sphere with $90^{\prime \prime}$ projected diameter. The model fit gives an area filling factor of 2.94 and, assuming a distance of $50 \mathrm{kpc}$, a volume filling factor of 0.001 . These area and volume filling factors imply that 
Table 4. Comparison between observed line integrated intensities and the predictions from the KOSMA- $\tau$ PDR model and the RADEX radiative transfer code.

\begin{tabular}{lccc}
\hline \hline Line & Observed & KOSMA- $^{a}$ & RADEX $^{b}$ \\
\hline${ }^{12} \mathrm{CO} J=1 \rightarrow 0$ & $2.8 \times 10^{-7}(0.2)$ & $8.7 \times 10^{-7}$ & $8.1 \times 10^{-7}$ \\
${ }^{12} \mathrm{CO} J=3 \rightarrow 2$ & $3.0 \times 10^{-5}(0.3)$ & $3.4 \times 10^{-5}$ & $3.2 \times 10^{-5}$ \\
${ }^{12} \mathrm{CO} J=4 \rightarrow 3$ & $7.8 \times 10^{-5}(1.6)$ & $7.8 \times 10^{-5}$ & $6.8 \times 10^{-5}$ \\
${ }^{12} \mathrm{CO} J=7 \rightarrow 6$ & $2.2 \times 10^{-4}(0.4)$ & $1.9 \times 10^{-4}$ & $2.1 \times 10^{-4}$ \\
${ }^{12} \mathrm{CO} J=9 \rightarrow 8$ & $<3.3 \times 10^{-4}$ & $9.4 \times 10^{-5}$ & $1.9 \times 10^{-4}$ \\
${ }^{13} \mathrm{CO} J=1 \rightarrow 0$ & $1.9 \times 10^{-8}(0.3)$ & $6.8 \times 10^{-8}$ & $3.1 \times 10^{-8}$ \\
${ }^{13} \mathrm{CO} J=4 \rightarrow 3$ & $1.0 \times 10^{-5}(0.3)$ & $1.1 \times 10^{-5}$ & $1.0 \times 10^{-5}$ \\
$\left.{ }^{[\mathrm{C}}\right]{ }^{3} \mathrm{P}_{1} \rightarrow{ }^{3} \mathrm{P}_{0}$ & $1.1 \times 10^{-5}(0.3)$ & $1.0 \times 10^{-5}$ & $5.5 \times 10^{-6}$ \\
${ }^{[\mathrm{C}}{ }^{3}{ }^{3} \mathrm{P}_{2} \rightarrow{ }^{3} \mathrm{P}_{1}$ & $4.1 \times 10^{-5}(1.7)$ & $4.4 \times 10^{-5}$ & $3.5 \times 10^{-5}$ \\
{$\left[\mathrm{C}_{\mathrm{II}}\right]{ }^{2} \mathrm{P}_{3 / 2} \rightarrow{ }^{2} \mathrm{P}_{1 / 2}$} & $1.2 \times 10^{-2}(0.01)$ & $2.4 \times 10^{-2}$ & $4.5 \times 10^{-3}$ \\
\hline
\end{tabular}

Notes. All intensities are in units of erg $\mathrm{cm}^{-2} \mathrm{~s}^{-1} \mathrm{sr}^{-1}$. The observed and RADEX intensities are corrected for beam dilution. The PDR model absolute intensities are calculated assuming a common FWHM source size of $90^{\prime \prime} .{ }^{(a)}$ Best-fit model: $n_{\mathrm{ens}}=3.3 \times 10^{5} \mathrm{~cm}^{-3}, M_{\mathrm{ens}}=1.2 \times$ $10^{5} M_{\odot}, \chi_{0}=3160{ }^{(b)}$ Best-fit model: $n_{\mathrm{H}_{2}}=10^{4} \mathrm{~cm}^{-3}, T_{\mathrm{kin}}=160 \mathrm{~K}$, beam filling $1 / 16, N_{\mathrm{CO}}=1.7 \times 10^{17} \mathrm{~cm}^{-2}, N_{\mathrm{C}^{0}}=5.6 \times 10^{16} \mathrm{~cm}^{-2}$.

the model requires many low-mass clumps to reproduce the observed line intensities. As discussed by e.g. Kramer et al. (2008), these low-mass clumps would require very high external thermal pressures to be confined and might be considered as transient features of the turbulent gas and should evaporate on short timescales. Note that the area filling factor defined here is not comparable with the beam filling factor derived in Sect. 4.2, because the PDR model does not predict whether these small clumps are clustered or dispersed within the cloud.

In Fig. 6 we show the dependence of the different observed transitions on different $\mathrm{H}_{2}$ volume densities and FUV radiation fields. Solutions in the range $n_{\mathrm{ens}}=1 \times 10^{5}-1 \times 10^{5.6} \mathrm{~cm}^{-3}$ and $\chi_{0}=400-6300$ are within three times the minimum $\chi_{\min }^{2}=59$. Note that this high value of $\chi^{2}$ is dominated by the inability of the PDR model to reproduce the [C II] emission. If we exclude the [C II] line in the calculation of $\chi^{2}$, we obtain a much lower $\chi_{\text {min }}^{2}$ of 0.01 , without significant changes in the constrained physical conditions.

To illustrate the importance of the observed line intensities in determining the value of $\chi_{0}$ and $n_{\text {ens }}$, we show in Fig. 7 the predicted model line intensities as a function of $n_{\text {ens }}$ for a fixed value of $\chi_{0}=3980$, and as a function of $\chi_{0}$ for a fixed value of $n_{\text {ens }}=3.9 \times 10^{5} \mathrm{~cm}^{-3}$. The fixed values of $\chi_{0}$ and $n_{\text {ens }}$ were selected to be the closest point in the model grid to the solution found using the simulated annealing technique. The intensity of the mid- $J{ }^{12} \mathrm{CO}$ and ${ }^{13} \mathrm{CO}$ are very sensitive to $n_{\text {ens }}$ and drive the value of this parameter. The $\left[\mathrm{C}_{\mathrm{I}}\right]$ and mid- $J \mathrm{CO}$ lines are sensitive to the FUV radiation field but to lesser extent than to $n_{\text {ens }}$. Consequently, as also seen in Fig. 6, the fit to the average ensemble volume density is better than that for the FUV radiation field. The decreasing value of the $\left[\mathrm{C}_{\mathrm{I}}\right]$ and mid- $J \mathrm{CO}$ lines and the increase of value of the $[\mathrm{C}$ II $]$ intensity with $\chi_{0}$ is related to the shift of the $\mathrm{C}^{+} / \mathrm{C}^{0} / \mathrm{CO}$ transition deeper into the cloud as $\chi_{0}$ increases, reducing the column density of $\mathrm{CO}$ and increasing column density of [C $\mathrm{II}$. As shown by Cubick et al. (2008), the emission from $\mathrm{CO}$ transitions with $J>5$ is mostly arising from low-mass clumps, while lower- $J \mathrm{CO}$ and $\left[\mathrm{C}_{\mathrm{I}}\right]$ transitions arise from clumps with larger masses. Therefore, the observed ${ }^{12} \mathrm{CO} J=7 \rightarrow 6$ transition is very sensitive to the lower mass limit and drives the value of this model parameter. For the upper mass limit, the dependence on the observed lines is weaker, and

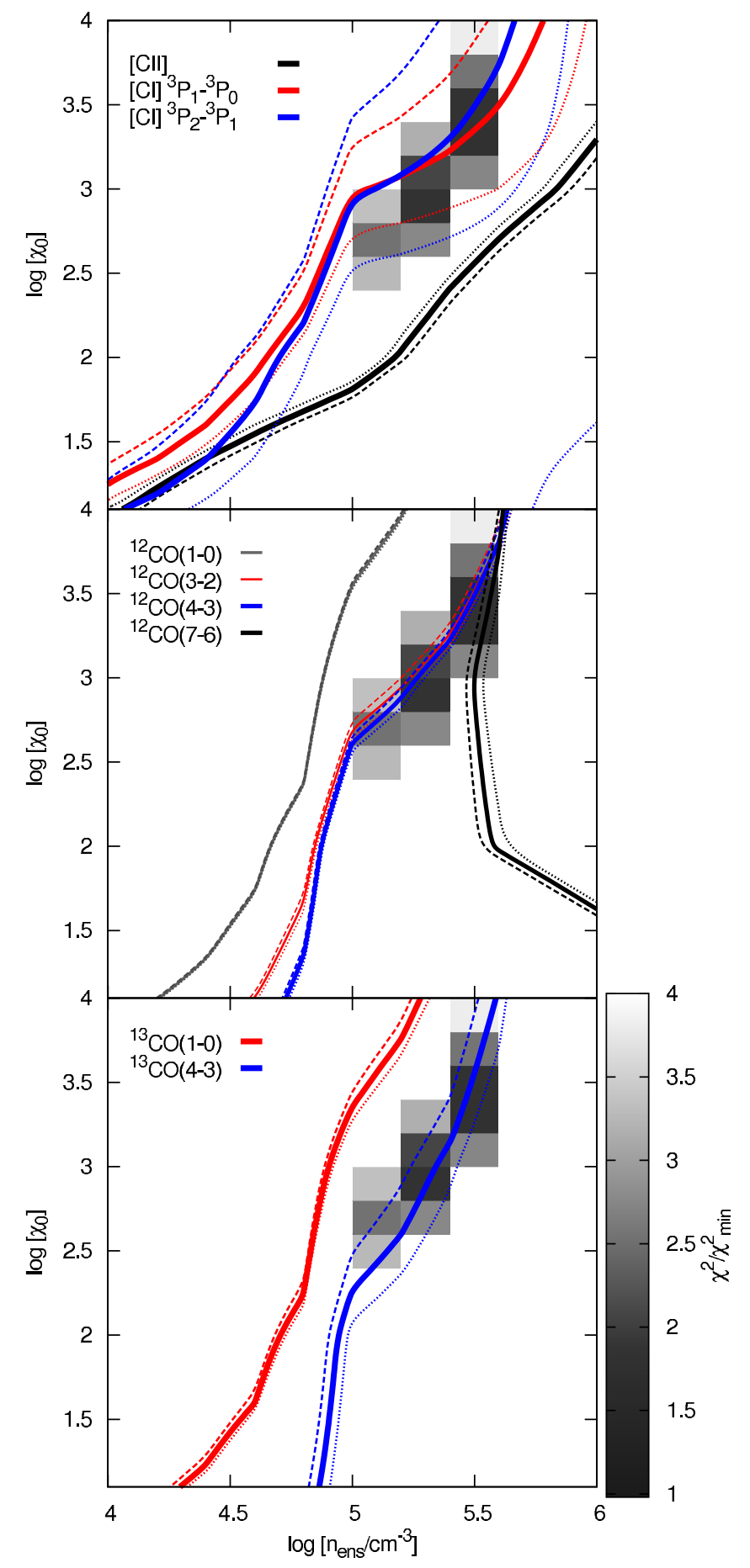

Fig. 6. Comparison between the different observed lines with the predictions from the clumpy-PDR model for a given $\mathrm{H}_{2}$ volume density and FUV radiation field. In all panels, the solid lines represent the observed values while the dashed and dotted lines represent their lower and upper error bars, respectively. The gray-scale represents the value of $\chi^{2}$ relative to its minimum value, $\chi_{\min }^{2}$.

varying the upper mass limit to higher values does not have a significant effect on the model fit.

In Fig. 6 and Table 4 (see also Fig. 8), we can see that the best-fit model agrees well with observations of mid- $J$ CO and $\left[\mathrm{C}_{\mathrm{I}}\right]$ but it overestimates the $\left[\mathrm{C}_{\mathrm{II}}\right],{ }^{12} \mathrm{CO} J=1 \rightarrow 0$, and ${ }^{13} \mathrm{CO} J=1 \rightarrow 0$ line intensities. To reproduce the lines observed by NANTEN2, the model requires clumps with volume densities that are much higher than the critical density of 

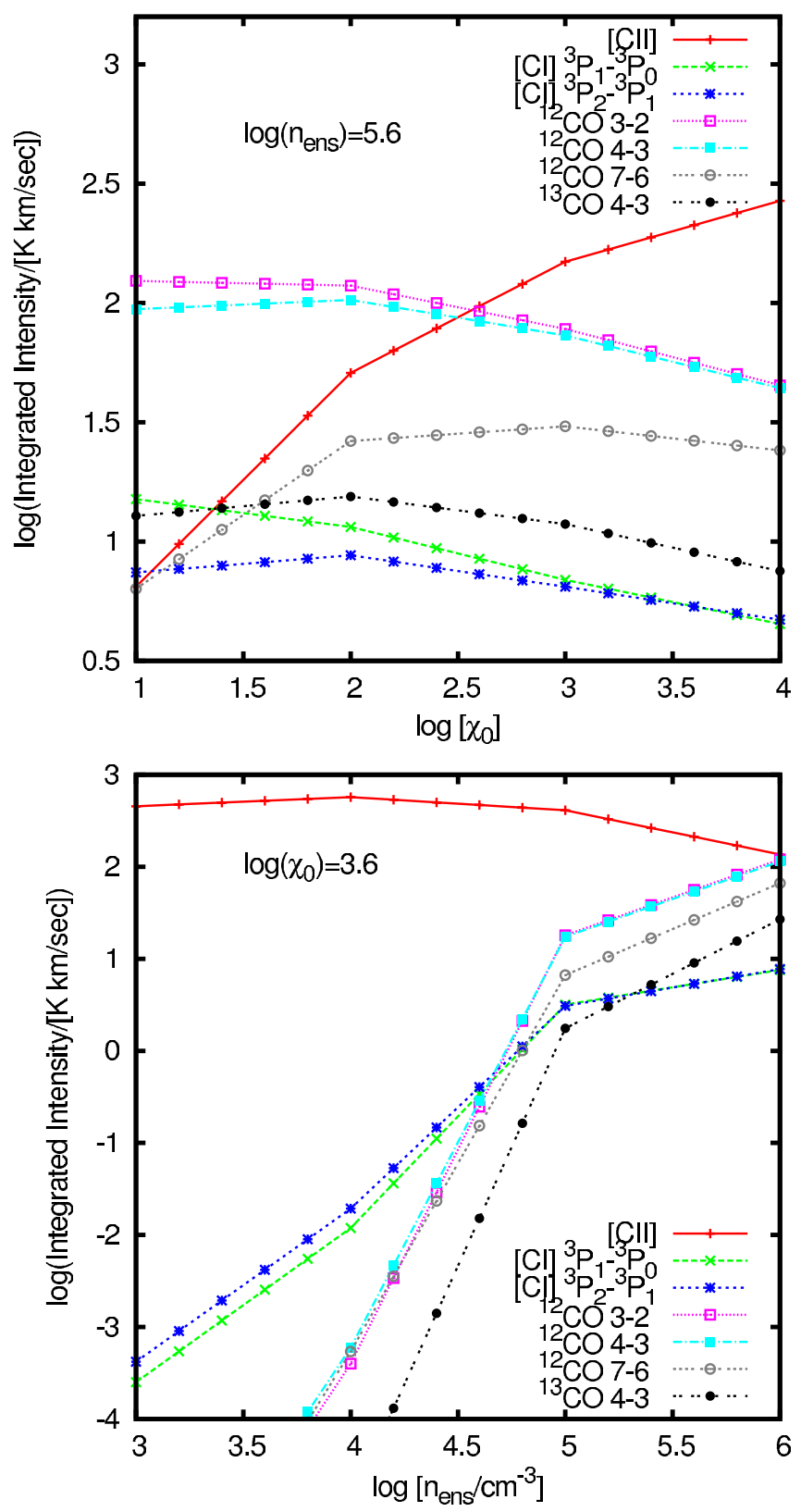

Fig. 7. Model-predicted integrated intensities the spectral lines used to constrain the PDR model as a function of $\chi_{0}$ for a fixed value of $n_{\text {ens }}$ (upper panel) and of $n_{\mathrm{ens}}$ for a fixed value of $\chi_{0}$ (lower panel).

the $[\mathrm{C}$ II $]$ line $\left(5 \times 10^{3} \mathrm{~cm}^{-3}\right.$; see Table 3$)$, resulting in a predicted $\left[\mathrm{C}_{\mathrm{II}}\right]$ intensity that is higher than observed. A model with a lower volume density might reproduce the $[\mathrm{C}$ II $]$ line better, but it would underestimate the mid- $J$ and $\left[\mathrm{C}_{\mathrm{I}}\right]$ transitions. Another complication is that, as shown in Fig. 1, we can expect that the FUV field is not uniform within 30 Dor-10, because parts of the cloud are closer to R136 than others, while the model assumes a uniform field. Note that we are only considering a single-component model in this analysis. It might be possible to find a better fit to the lines if more gas components were added, but more data (e.g. [OI] lines) would be needed to obtain a good constraint to the model. Note that, as mentioned above, the PDR model neglects any mutual absorption/shielding between clumps. Since the ${ }^{12} \mathrm{CO} J=1 \rightarrow 0$ is optically thick and ${ }^{13} \mathrm{CO} J=1 \rightarrow 0$ is likely affected by optical depth effects, radiation from background clumps should be (partly) shielded by foreground clumps. Because the model ignores this effect, it overestimates the intensity of these transitions. This effect might also partly be the reason for the overestimation of the [C $\mathrm{II}]$ line, because this line might be also affected by optical depth effects between clumps.

Our determination of the strength of the FUV field agrees excellently with the independent estimation presented by Poglitsch et al. (1995) of $\chi_{0}=3100-3600$. The constraints on the $\mathrm{H}_{2}$ volume density and FUV radiation field for 30 Dor are also consistent with previous determinations based on PDR modeling (Pak et al. 1998; Kaufman et al. 1999; Bolatto et al. 1999; Röllig et al. 2006). A crucial test to the suggested clumpy structure of the LMC's ISM will be high angular resolution imaging of low- and mid- $J$ CO and $\left[\mathrm{C}_{\mathrm{I}}\right]$ transitions with ALMA.

\section{Discussion}

\subsection{Comparison with LMC-N159W}

The 30 Dor-10 region is located at a distance of $\sim 20 \mathrm{pc}$ from the center of the R136 star cluster, hence this region is exposed to an extreme FUV radiation field. It is of interest to compare its physical properties with other regions in the LMC that have more moderate ambient radiation fields. Since there are no significant spatial variations of the metallicity in the LMC (Dufour 1984), this comparison will help us to understand the effect of the FUV radiation field on low-metallicity environments.

In Pineda et al. (2008) we made a similar analysis of the same set of spectral lines as analyzed here in the LMC-N159W region. We constrained the $\mathrm{H}_{2}$ volume density to be $\sim 10^{4} \mathrm{~cm}^{-3}$, a kinetic temperature of $\sim 80 \mathrm{~K}$ and equal $\mathrm{CO}$ and $\mathrm{C}_{\text {I }}$ column densities of $\sim 1.6 \times 10^{17} \mathrm{~cm}^{-2}$. The beam filling in $\mathrm{N} 159 \mathrm{~W}$ is about $1 / 6$. Modeling of the photon-dominated region suggests that the strength of the FUV field in N159W is about $\chi_{0}=220$, while we find $\chi_{0}=3160$ in 30 Dor-10 (Sect. 5).

The 30 Dor-10 region is warmer than N159W, with a kinetic temperature of about $160 \mathrm{~K}$ and $80 \mathrm{~K}$, respectively. This higher temperature is indicated by the higher ${ }^{12} \mathrm{CO} J=7 \rightarrow 6$ to $J=4 \rightarrow 3$ ratio in 30 Dor- 10 . This warming can be understood because the gas heating is likely dominated by photoelectric heating, which depends on the strength of the FUV field (Bakes \& Tielens 1998). The higher temperatures in 30 Dor-10 do not result in higher brightness temperatures compared with N159W because of the reduced beam filling (1/16). The column/volume densities, however, are similar or somewhat higher in 30 Dor- 10 compared with N159W, suggesting that CO clumps in the former region are smaller. These smaller clumps can be interpreted as the effect of the enhanced $\mathrm{CO}$ photo-destruction due to the strong FUV radiation field. This enhanced photodestruction does not result in a higher abundance of $\mathrm{C}$ I relative to $\mathrm{CO}$, becuase the $N(\mathrm{CO}) / N\left(\mathrm{C}_{\mathrm{I}}\right)$ column density ratio is even higher in 30 Dor-10, implying that the FUV field moves the $\mathrm{C}^{+} / \mathrm{C} / \mathrm{CO}$ transition layer to locations deeper into the clumps, but does not increase the thickness of this layer. Accordingly, most of the carbon in 30 Dor- 10 should be in the form of $\mathrm{C}^{+}$. This effect has been predicted by PDR models (e.g. Röllig et al. 2006). Note that we cannot eliminate other mechanisms that might destroy the molecular cloud, such as stellar winds from massive stars. However, the N159W should also be influenced by these effects.

We determined the column density of $\mathrm{C}^{+}$using the integrated intensity observed by Boreiko \& Betz (1991) of $7.7 \times 10^{-4} \mathrm{erg} \mathrm{cm}^{-2} \mathrm{~s}^{-1} \mathrm{sr}^{-1}$. This quantity is related to the $\mathrm{C}^{+}$column density, assuming a kinetic temperature larger than 


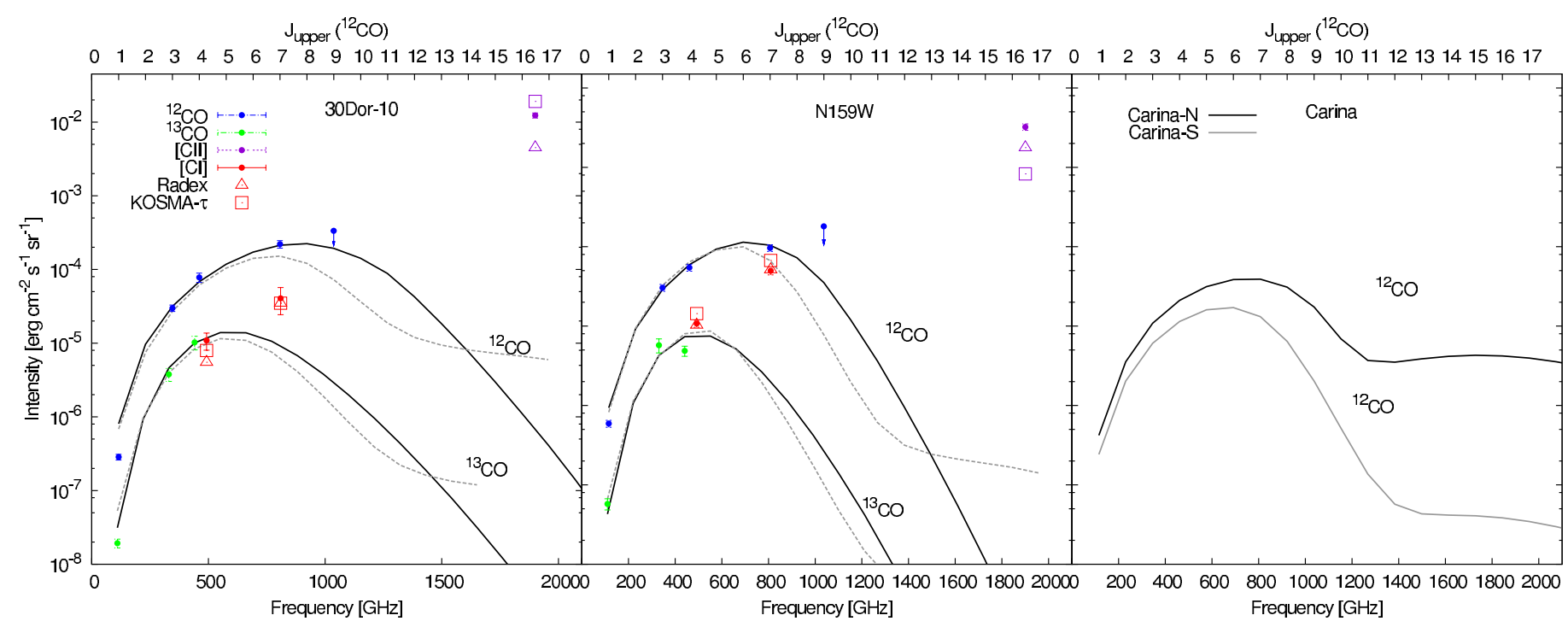

Fig. 8. Spectral-line energy distribution (SLED) for several millimeter and submillimeter lines toward 30 Dor-10 (left), N159W (center), and Carina-N and -S (right). In the left and central panel, the solid lines represent the predictions for the ${ }^{12} \mathrm{CO}$ and ${ }^{13} \mathrm{CO}$ SLED from the excitation analysis presented in Sect. 4 for 30 Dor-10 and in Pineda et al. (2008) for N159W. The gray-dashed lines represent the ${ }^{12}$ CO and ${ }^{13}$ CO SLED predicted by the clumpy PDR model fits. The excitation analysis and PDR model predictions for the [ $\left.\mathrm{C}_{\mathrm{I}}\right]$ and $\left[\mathrm{C}_{\mathrm{II}}\right]$ lines are represented by triangles and boxes, respectively. In the right panel we show the ${ }^{12} \mathrm{CO}$ SLED for the Carina-N and Carina-S regions presented by Kramer et al. (2008) using the same clumpy PDR model as used in this paper. The physical conditions derived from the PDR model are similar for 30 Dor-10 and Carina-N as well as for N159W and Carina-S, but they have different metallicity (Carina $Z=Z_{\odot} ; \mathrm{LMC} Z=0.4 Z_{\odot}$ ).

$91 \mathrm{~K}$ and a volume density higher than $n_{\mathrm{cr}}=5 \times 10^{3} \mathrm{~cm}^{-3}$, by $N\left(\mathrm{C}^{+}\right)=6.4 \times 10^{20} I\left(\left[\mathrm{C}_{\mathrm{II}}\right]\right) \mathrm{cm}^{-2}\left(\mathrm{erg} \mathrm{cm}^{-2} \mathrm{~s}^{-1} \mathrm{sr}^{-1}\right)^{-1}$ (Crawford et al. 1985). With that we obtain a column density of $\mathrm{C}^{+}$of $4.96 \times 10^{17} \mathrm{~cm}^{-2}$ and therefore the total carbon abundance in 30 Dor-10 is distributed as $N_{\mathrm{C}^{+}}: N_{\mathrm{C}}: N_{\mathrm{CO}}=$ 69\%:8\%:23\%. For comparison, the distribution of carbon in the $\mathrm{N} 159 \mathrm{~W}$ region is $46 \%: 27 \%: 27 \%$ while in the Galactic PDR DR21(OH) it is 3\%:10\%:87\% (Jakob et al. 2007). As in N159W, the 30 Dor-10 region has an increased fraction of the gas-phase carbon in the form of $\mathrm{C}^{+}$compared with DR21(OH).

\subsection{CO spectral-line energy distribution}

We combined our observed mid- $J{ }^{12} \mathrm{CO}$ observations with previously observed transitions to derive the $\mathrm{CO}$ spectral-line energy distribution (SLED) in 30 Dor-10 and N159W. We present the CO SLEDs in Fig. 8, together with the predictions from our excitation analyses and PDR modeling in both regions. We include observations of ${ }^{12} \mathrm{CO} J=3 \rightarrow 2$ from Minamidani et al. (2008) and upper limits to the intensity of the $J=9 \rightarrow 8$ line from Boreiko \& Betz (1991). We corrected these line intensities, together with that of the $J=7 \rightarrow 6$ transition, to match the intensity corresponding to a $38^{\prime \prime}$ beam (see Sect. 3 ). Singlecomponent models agree well with the observed $\mathrm{CO}$ transitions. But the model for 30 Dor-10 still predicts a line intensity of the $J=1 \rightarrow 0$ line that is about a factor of 3 higher than observed. The ${ }^{12} \mathrm{CO}$ SLED peaks at $J=8 \rightarrow 7$ for 30 Dor-10 and $J=6 \rightarrow 5$ for N159W. The different CO excitation in both regions reflects the difference in their kinetic temperature, which in turn is due to the difference in the FUV radiation field between both regions.

The CO SLED predicted by the clumpy PDR model for 30 Dor-10 is similar to that predicted by the excitation analysis for low- $J$ transitions, but differs significantly for high- $J$ transitions, with the PDR model predicting much higher intensities for $J>6$. For $J>14$, however, the PDR model predicts almost constant intensities, while the intensity predicted by the excitation model drops. The clumpy PDR model predicts a peak of the CO SLED at a lower ${ }^{12} \mathrm{CO}$ transition $(J=$ $6 \rightarrow 5$ ) compared with the prediction from the excitation analysis $(J=8 \rightarrow 7)$. For N159W the clumpy PDR model CO SLED also predicts lower intensities for high- $J$ transitions but the difference is smaller than for 30 Dor-10. The peak of the CO SLED still coincides with that predicted by the excitation analysis $\left({ }^{12} \mathrm{CO} J=6 \rightarrow 5\right)$. More observations of high- $J$ CO transitions with e.g. Herschel or SOFIA in 30 Dor-10 and N159W are required to test the predictions of the PDR modeling and excitation analysis for high- $J$ CO transitions.

In Fig. 8 we also show the CO SLED for two positions in the Carina region, Carina- $\mathrm{N}$ and Carina-S, whose physical conditions have been constrained with the KOSMA- $\tau$ PDR model by Kramer et al. (2008) using the same set of line as presented here. The constrained values of $\left(\chi_{0}, n_{\text {ens }}\right)$ are $\left(3100,2 \times 10^{5} \mathrm{~cm}^{-3}\right)$ for Carina-N and $\left(310,2 \times 10^{5} \mathrm{~cm}^{-3}\right)$ for Carina-S. Therefore, both Carina-N and Carina-S have similar physical conditions compared with 30 Dor-10 and N159W, respectively, but solar metallicity. The metallicity, however, seems not to affect the shape of the CO SLED because for both pairs of sources the CO spectral energy distribution is consistent within the small differences in the physical conditions and uncertainties of the models.

The total CO luminosity $\left(L_{\mathrm{CO}}\right)$ predicted by the radiative transfer code in an area defined by the $38^{\prime \prime}$ beam of our NANTEN2 observations (corresponding to $9.5 \mathrm{pc}$ at a distance of $50 \mathrm{kpc}$ ) is $1.1 \times 10^{36} \mathrm{erg} \mathrm{s}^{-1}$ for 30 Dor-10 and $4.3 \times$ $10^{35} \mathrm{erg} \mathrm{s}^{-1}$ for N159W. The clumpy PDR model predicts a somewhat lower $L_{\mathrm{CO}}$ of $8.2 \times 10^{35} \mathrm{erg} \mathrm{s}^{-1}$ for 30 Dor-10 and $2.5 \times 10^{35} \mathrm{erg} \mathrm{s}^{-1}$ for N159W. From the observations, we also estimated the total $\left[\mathrm{C}_{\mathrm{II}}\right]$ and $\left[\mathrm{C}_{\mathrm{I}}\right]$ luminosity (ignoring the much weaker $\left[\mathrm{CI}_{\mathrm{I}}\right]{ }^{3} \mathrm{P}_{2} \rightarrow{ }^{3} \mathrm{P}_{0}$ transition) of $9.9 \times 10^{36}$ and $4.2 \times 10^{34} \mathrm{erg} \mathrm{s}^{-1}$, respectively for 30 Dor-10 and $2.6 \times 10^{36}$ and $4.9 \times 10^{34} \mathrm{erg} \mathrm{s}^{-1}$, respectively, for $\mathrm{N} 159 \mathrm{~W}$. The relative fraction of the luminosity of these species is $L_{[\mathrm{C} \text { II] }}: L_{[\mathrm{CI}]}: L_{\mathrm{CO}}=$ 90\%:0.4\%:9.6\% for 30 Dor-10 and 84.4\%:1.6\%:14 for N159W, when using $L_{\mathrm{CO}}$ predicted by the radiative transfer code, and 
92\%:0.4\%:7.6\% for 30 Dor-10 and 90\%:1.7\%:8.3\% for N159W, when using $L_{\mathrm{CO}}$ predicted by the clumpy PDR model. We also estimates a total [O I] luminosity of $7.4 \times 10^{36} \mathrm{erg} \mathrm{s}^{-1}$ from observations of the [O I] $63 \mu \mathrm{m}$ and $146 \mu \mathrm{m}$ lines by Poglitsch et al. (1995) in 30 Dor. These estimated luminosities suggest that [C II] and [ $\mathrm{O}_{\mathrm{I}}$ ] contribute equally to the gas cooling in 30Dor-10. This significant contribution of $\left[\mathrm{O}_{\mathrm{I}}\right]$ to the line cooling confirms the warm and dense gas conditions derived with the excitation analysis and PDR modeling. Note that we cannot rule out the possibility of an additional component powering even higher $\mathrm{CO}$ transitions $(J>10)$ in 30 Dor-10 and N159W. Therefore, our estimate of the total CO luminosity might be a lower limit. As mentioned above more observations will allow us to determine the contribution of high- $J$ CO transitions to the total CO luminosity.

\section{Summary and conclusions}

We derived the physical conditions of the line-emitting gas in the 30 Dor-10 region in the LMC. We compared an excitation/radiative transfer code and a PDR model with NANTEN2 observations of the ${ }^{12} \mathrm{CO} J=4 \rightarrow 3, J=7 \rightarrow 6$, and ${ }^{13} \mathrm{CO} J=4 \rightarrow 3$ rotational and $\left[\mathrm{C}_{\mathrm{I}}\right]{ }^{3} \mathrm{P}_{1}-{ }^{3} \mathrm{P}_{0}$ and ${ }^{3} \mathrm{P}_{2}-{ }^{3} \mathrm{P}_{1}$ finestructure transitions. Our results can be summarized as follows:

- The analysis of the excitation conditions for both the CO submm-lines and the $\left[\mathrm{CI}_{\mathrm{I}}\right]$ fine structure lines shows temperatures of about $T=160 \mathrm{~K}$ and densities of about $n_{\mathrm{H}_{2}}=$ $10^{4} \mathrm{~cm}^{-3}$ for the emitting gas.

- We found that 30 Dor-10 is warmer and has a lower beam filling factor compared to N159W. This difference might be the result of the FUV radiation field heating the gas and photodissociating CO molecules. This, however, does not result in an enhanced $\mathrm{C}$ abundance relative to $\mathrm{CO}$ : we obtained a $N(\mathrm{CO}) / N\left(\mathrm{C}_{\mathrm{I}}\right)$ ratio of 3 in 30 Dor-10, which is higher than that found in $\mathrm{N} 159 \mathrm{~W}$ of $N(\mathrm{CO}) / N\left(\mathrm{C}_{\mathrm{I}}\right)$ equal to unity.

- We derived the CO spectral-line energy distribution of 30 Dor-10 and N159W. Considering the excitation conditions constrained using line ratios, we found that this distribution peaks at ${ }^{12} \mathrm{CO} J=8 \rightarrow 7$ for 30 Dor-10 and at $J=6 \rightarrow 5$ in N159W. The clumpy PDR model, however, predicts the peak of the $\mathrm{CO}$ spectral-line energy distribution at $J=6 \rightarrow 5$ for both 30 Dor-10 and N159W.

- We compared our observations with the results of a clumpy PDR model. The model that best reproduces the observed absolute integrated intensities has an average ensemble $\mathrm{H}$ density of $n_{\mathrm{ens}} \sim 10^{5} \mathrm{~cm}^{-3}$, a total mass of the ensemble, $M_{\mathrm{ens}} \sim$ $10^{5} \mathrm{M}_{\odot}$, and a strength of the FUV field of $\chi_{0} \sim 3100$. The constraints on the $\mathrm{H}_{2}$ volume density and the strength FUV radiation field agree well with independent determinations and previous PDR modeling results.

The 30 Dorarus region is the closest example of vigorous star-formation taking place in a low-metallicity environment, which is thought to be common in the early universe. Our results suggest that the star-forming ISM in 30 Dor-10, and perhaps in starburst galaxies in the early universe, is dense, and warm, and clumpy. The clumpy structure of the 30 Dor- 10 gas is a result of the extreme FUV environment to which the low-metallicity gas is exposed. Our results also highlight the importance of ionized carbon in tracing the total gas mass and, together with atomic oxygen, in regulating the thermal balance of the gas. Future observations of fine-structure transitions of [ $\left.\mathrm{C}_{\mathrm{II}}\right],\left[\mathrm{O}_{\mathrm{I}}\right.$,
$[\mathrm{NII}]$, and $\left[\mathrm{C}_{\mathrm{I}}\right]$ and rotational transitions of $\mathrm{CO}$ in the local Universe are essential for our understanding of star-formation in low-metallicity environments and are an important tool for the interpretation of observations of high-redshift galaxies with ALMA.

Acknowledgements. This research was conducted at the Jet Propulsion Laboratory, California Institute of Technology under contract with the National Aeronautics and Space Administration. We would like to thank William Langer and Paul Goldsmith for careful reading of the manuscript and enlightening discussions. The NANTEN2 project (southern submillimeter observatory consisting of a 4-m telescope) is based on a mutual agreement between Nagoya University and The University of Chile and includes member universities from six countries, Australia, Republic of Chile, Federal Republic of Germany, Japan, Republic of Korea, and Swiss Confederation. M.R. wishes to acknowledge support from FONDECYT(CHILE) grant 1080335.

\section{References}

Bakes, E. L. O., \& Tielens, A. G. G. M. 1998, ApJ, 499, 258

Bolatto, A. D., Jackson, J. M., \& Ingalls, J. G. 1999, ApJ, 513, 275

Boreiko, R. T., \& Betz, A. L. 1991, ApJ, 380, L27

Chen, C.-H. R., Indebetouw, R., Chu, Y.-H., et al. 2010, ApJ, 721, 1206

Crawford, M. K., Genzel, R., Townes, C. H., \& Watson, D. M. 1985, ApJ, 291, 755

Cubick, M., Stutzki, J., Ossenkopf, V., Kramer, C., \& Röllig, M. 2008, A\&A, 488,623

Draine, B. T. 1978, ApJS, 36, 595

Dufour, R. J. 1984, in Structure and Evolution of the Magellanic Clouds, eds. S. van den Bergh, \& K. S. D. Boer, IAU Symp., 108, 353

Dufour, R. J., Shields, G. A., \& Talbot, Jr., R. J. 1982, ApJ, 252, 461

Feast, M. 1999, PASP, 111, 775

Fukui, Y., Kawamura, A., Minamidani, T., et al. 2008, ApJS, 178, 56 Grenier, I. A., Casandjian, J.-M., \& Terrier, R. 2005, Science, 307, 1292 Heikkilä, A., Johansson, L. E. B., \& Olofsson, H. 1999, A\&A, 344, 817 Hollenbach, D. J., \& Tielens, A. G. G. M. 1999, Rev. Mod. Phys., 71, 173 Hughes, A., Wong, T., Ott, J., et al. 2010, MNRAS, 406, 2065

Israel, F. P. 1997, A\&A, 328, 471

Jakob, H., Kramer, C., Simon, R., et al. 2007, A\&A, 461, 999

Johansson, L. E. B., Greve, A., Booth, R. S., et al. 1998, A\&A, 331, 857

Kaufman, M. J., Wolfire, M. G., Hollenbach, D. J., \& Luhman, M. L. 1999, ApJ, 527, 795

Kim, S. 2006, PASP, 118, 94

Kim, S., Walsh, W., Xiao, K., \& Lane, A. P. 2005, AJ, 130, 1635

Kramer, C., Cubick, M., Röllig, M., et al. 2008, A\&A, 477, 547

Langer, W. D., Velusamy, T., Pineda, J. L., et al. 2010, A\&A, 521, L17

Madden, S. C., Poglitsch, A., Geis, N., Stacey, G. J., \& Townes, C. H. 1997, ApJ, 483, 200

Meixner, M., Gordon, K. D., Indebetouw, R., et al. 2006, AJ, 132, 2268

Minamidani, T., Mizuno, N., Mizuno, Y., et al. 2008, ApJS, 175, 485

Minamidani, T., Tanaka, T., Mizuno, Y., et al. 2011, AJ, 141, 73

Pak, S., Jaffe, D. T., van Dishoeck, E. F., Johansson, L. E. B., \& Booth, R. S. 1998, ApJ, 498, 735

Pineda, J. L., Mizuno, N., Stutzki, J., et al. 2008, A\&A, 482, 197

Pineda, J. L., Ott, J., Klein, U., et al. 2009, ApJ, 703, 736

Planck Collaboration 2011, A\&A, 536, A19

Poglitsch, A., Krabbe, A., Madden, S. C., et al. 1995, ApJ, 454, 293

Röllig, M., Ossenkopf, V., Jeyakumar, S., Stutzki, J., \& Sternberg, A. 2006, A\&A, 451, 917

Schöier, F. L., van der Tak, F. F. S., van Dishoeck, E. F., \& Black, J. H. 2005, A\&A, 432, 369

Stark, A. A., Bolatto, A. D., Chamberlin, R. A., et al. 1997, ApJ, 480, L59

Störzer, H., Stutzki, J., \& Sternberg, A. 1996, A\&A, 310, 592

Tielens, A. G. G. M., \& Hollenbach, D. 1985, ApJ, 291, 722

van der Marel, R. P., \& Cioni, M.-R. L. 2001, AJ, 122, 1807

van der Tak, F. F. S., Black, J. H., Schöier, F. L., Jansen, D. J., \& van Dishoeck, E. F. 2007, A\&A, 468, 627

Velusamy, T., Langer, W. D., Pineda, J. L., et al. 2010, A\&A, 521, L18

Werner, M. W., Becklin, E. E., Gatley, I., et al. 1978, MNRAS, 184, 365

Westerlund, B. E. 1997, The Magellanic Clouds (New York: Cambridge Univ. Press)

Wolfire, M. G., Hollenbach, D., \& McKee, C. F. 2010, ApJ, 716, 1191

Wong, T., Hughes, A., Ott, J., et al. 2011, ApJS, 197, 16 